\title{
Generisch-serielle Re-Lektüren in Psycho II-IV zwischen Interdependenzen und Slashergenre
}

Nur drei Jahre nach Hitchcocks Tod erscheint 1983 mit Psycho II unter der Regie von Richard Franklin die erste Fortsetzung von Psycho, auf die in nur kurzen Abständen 1986 und 1990 zwei weitere folgen: Psycho III und Psycho IV - The Beginning. Die drei Sequels folgen dabei einer populären Welle an Horrorfortsetzungen, die die Geschichten ihrer Vorgängerproduktionen ebenso erfolgreich fortführen. It Lives Again (1978), Damien: Omen II (1978), Dawn of the Dead (1978), Friday the 13th Part 2 (1981) und Halloween II (1981), um nur einige zu nennen, markieren in den 1970er und frühen 1980er Jahren den seriellen Auftakt eines Genretrends, der sich bis in die Gegenwart zieht. ${ }^{1}$ Auch Psycho II-IV folgen diesem Genretrend, greifen aber nicht irgendeinen erfolgreichen Einzelfilm auf, sondern mit „Hitchcock’s 1960 classic“ (Canby) einen zur Genreikone gewordenen Horrorfilm, wie die Filmkritiken zu Psycho II betonen: „To spin off Hitchcock's Psycho isn't irreverent. After all, [...] it is a brilliant piece of manipulative movie-making, set forever apart from all other horror films." (Ebd.) Mit Hitchcocks berüchtigter Geschichte über den jungen Motelbesitzer Norman Bates und sein mörderisches Alter Ego „Mother“ führen die Sequels die im Horrorgenre erstmals (neben dem britischen, aber weniger populären Peeping Tom) angebotene Semantik eines mörderischen Wahnsinns fort. ${ }^{2}$ Während die meisten Horrorfortsetzungen der damaligen Zeit nur wenige Jahre nach ihrer ersten Erzählung ansetzen und ihre Serienmörderfiguren unbeirrbar weitermorden lassen, können die Sequels zwei Jahrzehnte später nicht, einfach` nahtlos an Psycho

\footnotetext{
${ }^{1} \mathrm{Zu}$ genrespezifischen Modernisierungstrends von Horrorsequels siehe Abschn. 1.2.

${ }^{2}$ Die Schreibweise von „Mother“ bezieht sich auf Normans psychopathologische Imagination und Verkörperung seiner (toten) Mutter als lebendige und mit ihm interagierende Persönlichkeit hin. Die doppelten Anführungszeichen referieren auf Normans Anrufung seiner Mutter als „Mother“.
} 
anschließen und Norman Bates in sein altes Setting heimkommen lassen. ${ }^{3}$ Für die Fortsetzung seiner Taten müssen ,neue' Erklärungen gefunden werden, um die lange ,mordfreie' Zeitspanne und die seitdem stattgefundenen genrehistorischen Veränderungen zu überbrücken. Seit Psycho hat das Horrorgenre mit den populären Slasherfilmen vor allem ab Mitte der 1970er Jahre einen entscheidenden ästhetischen Zugewinn an Gewaltdarstellungen erfahren, die das Zeigen expliziter Schnitte, blutiger Wunden und massiver Körperzerstörungen zur Konvention machen. ${ }^{4}$ In Hitchcocks Psycho entdeckt Clover hierbei den ,ancestor“ (,Men, Women“ 23) des Slasherfilms, der mehrere neue Muster in das Horrorgenre eingeführt hat, die schließlich in den Filmen der 1970er und 1980er Jahre an Explizitheit gewinnen. 23 Jahre später führen die Psycho-Sequels damit das Genre an seine Anfänge zurück und überführen den eigenen Vorläufer in die gegenwärtige Genreform eines Slasherfilms, in der Norman Bates (vorerst) kein Serienmörder mehr ist, sondern geheilt aus der Heilanstalt zurückkehrt. ${ }^{5}$

Über die titelspezifische Markierung und serielle Fortführung der als abgeschlossen geltenden Erzählung eröffnet sich in den Sequels ein Feld wechselseitiger Bezugsverhältnisse, in denen nicht nur die Fortsetzungen in Bezug zu Psycho gesetzt werden, sondern auch Psycho durch die Fortsetzungen retrospektiv neue Bezüge erhält. Im Kontext des populären Slasherfilms der 1980er Jahre und seiner neuen Darstellungskonventionen führen diese intertextuellen Bezüge zu vergleichenden Genrelektüren. Die Sequels halten für das Publikum der 1980er Jahre - so die Ausgangsthese dieses Kapitels - weniger eine reine Fortsetzungsgeschichte als vielmehr eine sich über drei Produktionen erstreckende spezifische Horrorversion bereit, die Psycho und dessen Genremerkmale zur nachträglichen Lektüre anbieten und zugleich eine aktualisierte und generationenübergreifende Genreversion von Psycho hervorbringen. ${ }^{6}$ Die Sequels bilden

\footnotetext{
${ }^{3}$ Die Kulisse aus dem viktorianischen Haus auf dem Hügel und dem etwas schäbigen alten Motel ist inzwischen zu einer beliebten Touristenattraktion der Universal Studiotouren geworden (Canby).

${ }^{4}$ In Europa finden sich mit dem italienischen Giallo bereits seit den 1960er Jahren frühe Varianten des Slashers, in denen junge Frauen auf brutale Weise von maskierten Serienmördern getötet werden (siehe Scheinpflug, insbesondere 76).

${ }^{5}$ Die gegenwärtige Genreform eines Slasherfilms bezieht sich auf das semantische Genrekonzept und damit auf alle drei Sequels, die als ein ,exklusives' Genrekorpus betrachtet werden.

${ }^{6}$ In Rückgriff auf Aleida und Jan Assmanns Begriff des kulturellen Gedächtnisses erlöschen Generationengedächtnisse nach 30 Jahren. Die Sequels bieten damit kurz vor dem Ende der Psycho-Generation eine neue Version, die beide zusammenführt und zugleich im Sinne von Aleida und Jan Assmann als ,externalisiertes Gedächtnis“ funktioniert. Zum kulturellen Gedächtnis siehe A. Assmann und J. Assmann.
} 
vor diesem Hintergrund spezifische Genreausprägungen ihrer Zeit. Indem sie die mit einem vertrauten Narrativ einhergehenden Figuren, Orte und Szenen aufgreifen und den genrespezifischen Darstellungskonventionen anpassen, bewegen sich die Fortsetzungen zwischen intertextuellen Interdependenzen und generischen Semantiken des Slasherfilms und gehen in generisch-seriellen Wechselverhältnissen auf. Durch ihre enge Produktionsphase von sieben Jahren formieren die Sequels eine generische Zykluseinheit im Anschluss an den „Cycle“-Ansatz von Leger Grindon, der sich als ,exklusives ' Korpus für eine historische Genreanalyse auf der Ebene des Genrekonzepts eignet. ${ }^{7}$ Zyklen kennzeichnen innerhalb einer begrenzten Zeitspanne engmaschige Iterationen, weshalb Grindon Zyklen als ,a distinctive and more focused category“ (44) ansieht, die einen geeigneten Rahmen für historische Analysen von generischen Iterationsmustern bereithalten: „Furthermore, historical analysis of film genres benefits from a sharp focus on cycles of productions, as a focus on cycles responds to the critical call to emphasize the breaks and discontinuities in genre history rather than a smooth evolution." (Ebd. 45) Um die Kontinuitäten und Variationen der Iterationsmuster in den Sequels analytisch herauszuarbeiten, wird zuerst ein intertextueller Genreansatz in Rückgriff auf Ansätze zu Remakes und Serialität erarbeitet, um die interdependenten Verhältnisse zwischen Vor- und Nachgängerfilmen in ihrer retrospektiven Serialisierung sowie ihren wechselseitigen Relationierungen zu erarbeiten. Hierbei wird sich kritisch mit der Differenz zwischen, Original' und ,Kopie' auseinandergesetzt, um die der ,primären' Vorgängerproduktion häufig zugeschriebene Originalität zu dekonstruieren und die als Nachahmungen deklassierten , sekundären“ Fortsetzungen aufzuwerten. Über die Auflösung der (vermeintlichen) linearen Anordnungsverhältnisse wird eine offene Intertextualität erarbeitet, die die Psycho-Fortsetzungen als ein generisch-serielles Aushandlungsfeld beschreibbar macht und die Grundlage für die anschließende Analyse der Iterationsmuster bildet. In einem ersten analytischen Fokus wird die Wiederaufnahme bekannter Figuren und Orte in ihren ästhetischen Variationen untersucht, bevor sich in einem zweiten Schritt der Duschszene gewidmet wird. Diese wird einerseits als Psycho-Zitat eingebaut und anderseits in eigenen Versionen dekonstruiert, sodass sich selbstreflexive , Re-Lektüren ${ }^{\circ} 8$ ergeben, die die Duschszene als ,historische" Genresignatur festschreiben und ein Vergleich mit den ,neuen“ Tötungsszenen forcieren. Diese werden in einem letzten Schritt mithilfe der Ansätze von Carol J. Clover zum Slashergenre (,Men, Women“) und von

\footnotetext{
${ }^{7}$ Zum Zyklen-Ansatz siehe auch Abschn. 2.3.3.

${ }^{8}$ Der Sprachgebrauch von ,Re-Lektüren' umfasst sowohl die Lektüre der Sequels in Bezug zu Psycho als auch die Relektüre von Psycho unter der Perspektive der Sequels.
} 
Linda Williams zum Horrorfilm („Film Bodies“) analysiert. Clover und Williams Theorien zum modernen Horrorfilm bilden den geeigneten semantischen Rahmen, um die Tötungsszenen als ,neue' Signaturen des Schreckens zu erarbeiten. ${ }^{9}$ Clovers Ansatz operiert auf der für dieses Kapitel nötigen Ebene des Genrekonzepts und erarbeitet ihre Genretheorie entlang von Slasherfilmen, die wie die ersten beiden Psycho-Sequels zwischen 1974 und 1986 entstanden sind. Ihr intertextuell weit gefasstes Genreverständnis begreift Horrorfilme als stete Variationen und schematisch-repetitive Formen, wie es für die folgende Analyse der Sequels dienlich ist. Zudem bietet sie für die Analyse der ,neuen' Tötungsszenen drei produktive Bezugspunkte: Waffen, Opfer und Schocks (,Men, Women“ 23-42). Ihre Ausführungen werden von Williams' Ansatz der body genres unterfüttert, um die Darstellung exzessiver Körperlichkeit hinsichtlich ihrer Affektökonomien zu verdeutlichen. Ziel der Analysen ist es, Differenzverfahren herauszuarbeiten, die sowohl ,historische" als auch neue Genremerkmale in generisch-serielle Bezugsverhältnisse übergehen lassen.

\subsection{Zu Interdependenzen in Fortsetzungen}

Fortsetzungen referieren durch die zunehmende Zählweise im Titel auf ihre vorherigen wie potentiell nachfolgenden Produktionen. Sie stellen Verfahren dar, die im filmischen Material den Status des Vorgefundenen einziehen und dieses als Aneignung, Wiederaufnahme und Fortführung ausstellen. Dabei entstehen Unterteilungen zwischen einem primären Vorgängerfilm, der zum ,originären Ausgangstext ${ }^{\star}$ deklariert wird, und einer sekundären Nachfolgeproduktion. Frank Kelleter betont in dem Zusammenhang, dass die Ausgangstexte häufig als abgeschlossene Werke angelegt und vor allem in ihren Alleinstellungsmerkmalen vermarktet werden, sodass sie erst im Prozess der Wiederaufnahme oder auch des Remakings geöffnet und fortsetzbar gemacht werden (,Remake“ 155). Dies leistet Psycho II sowohl in Rückbezug auf Psycho als auch in Prospektion für die gesamte Sequel-Reihe.

Für Kelleter findet im Prozess der Fortsetzung eine rückwirkende Kanonisierung der Ausgangstexte als ,Originale“ oder auch ,Klassiker“ statt, was dazu führt, dass ,die zunächst unverbundenen, Versionen “ ein und desselben filmischen Narrativs retrospektiv auf einer höheren Ebene cinematischer Selbsthistorisierung serialisiert (werden)“ (ebd. 155). Das Serielle dieser Filmreihen folgt, wie

\footnotetext{
${ }^{9} \mathrm{Zu}$ den Konzepten von Clover und Williams siehe Abschn. 2.1.2.
} 
Michaela Wünsch als Unterschied zur Serie bemerkt, nicht zwingend einer linearen Anordnung, die die Geschichte kontinuierlich forterzählt, sondern umfasst ebenso Remakes, die die Geschichte neu erzählen, oder auch Prequels, deren Handlung vor der Geschichte des ,ersten ' Films angesiedelt sind (5). ${ }^{10}$ Nach Kelleter verbindet die so entstehende retrospektive Serialisierung die kommerziellen Interessen an verkürzten Herstellungszeiten und finanzieller Risikominimierung mit narrativen Eigenschaften der Selbstreferenz und einer nachträglichen Denomination, die sowohl mediales Wissen über einzelne Geschichten als auch deren Variationsbreite organisieren (,Remake“ 156 ff.). In der Rezeption werden damit populärkulturelle Referenzen immer wichtiger, weil sie die Dechiffrierung zu einem zunehmenden Unterhaltungsmehrwert generieren und cinephile Expertise erzeugen, sodass sich filmhistorisches Mehrwissen beim Publikum anreichert. Dies ist nicht nur aus einer kulturgeschichtlichen und medienhistorischen Perspektive relevant, sondern auch genrehistorisch überaus bedeutsam. Der Bestand populärer Erzählungen wird nach Kelleter nicht nur über filmisches Remaking gesichert, sondern auch rückwirkend mitbestimmt, weil in das historische Selbstverständnis durch die Filme selbst eingegriffen wird (,Remake“ 159 f.). Dieser Eingriff in die eigene Historiografie gilt für Sequels wie Genres, da beide ,vertraute Figurenkonstellationen und Handlungsabläufe wiederaufnehmen und in neue (mediale, politische, mentalitätsgeschichtliche usw.) Kontexte stellen, deren Aktualität gerade durch die Vertrautheit der narrativen Welt akzentuiert und reflektiert wird“" (ebd. 161). Das hat für die Betrachtungen generischer Zusammenhänge und Verschiebungen in den Sequels aus mehreren Gründen besondere Gültigkeit. Die serielle Wiederkehr bekannter Figuren und Handlungen erzeugt nicht nur eine narrative Vertrautheit im Sinne Kelleters; die Psycho-Sequels erzeugen durch die kontinuierliche Verkörperung von Anthony Perkins und die wiederkehrenden Orte auch eine ästhetische Vertrautheit. Über die Einbettung in Semantiken des Slasherfilms werden die Figuren und Orte zu neuen Genreformen, die in ihrer Variation einen kontrastreichen Bezug zu Psycho beibehalten. Die Konfrontation verläuft also wechselseitig und sorgt dafür, dass sich die bekannten Figuren, Orte und Szenen allmählich aus dem Einfluss von Hitchcocks Psycho lösen und in ein ,neues Leben ' im Generisch-Seriellen der Horror-Sequels eintreten. Für dieses neue Leben ist es zuträglich, das von Kelleter zuvor angedeutete Verhältnis zwischen ,Original ' und dem ,Nachfolger" einer kritischen Befragung zu unterziehen.

\footnotetext{
${ }^{10}$ Wobei auch Serien diese lineare Ordnung regelmäßig aufbrechen und mehrere Zeitebenen parallel erzählen.
} 
Gisela Fehrmann et al. diskutieren dieses Verhältnis im Rahmen von Praktiken des Sekundären. Zwischen dem vermeintlichen ,Original ' und der nachfolgenden ,Kopie' beobachten Fehrmann et al. eine metaleptische Umkehrung von Ursache und Wirkung, die den Status des Originals erst herbeiführt und ausweist.

Die Beziehung zwischen Original und Kopie lässt sich in dieser Hinsicht als eine Form von metaleptischer Umkehrung der Ursache-Wirkung-Relation beschreiben, bei der das vermeintlich primäre Original über Praktiken der Wiederholung und der Wiederaufnahme erst nachträglich als originär ausgewiesen wird - und zwar dadurch, dass diese Praktiken sich selbst als sekundäre Verfahren zu erkennen geben. (9f.)

Die Beziehung zwischen ,Original' und ,Kopie‘, so Fehrmann et al. weiter, stellt sich nicht als verquere Konstellation dar, sondern als widersprüchliche Anordnung. Denn das vermeintliche Original generiert sich durch eine Praxis, die der Idee des singulären Originals zuwider laufen müsste, aber stattdessen seinen Status potenziert, weil sich die angebliche Kopie selbst eine Zweitrangigkeit attestiert. Die Unterscheidung zwischen Original und Kopie ist demnach das Ergebnis von Produktionsprozessen, die nach Fehrmann et al. über „Transkriptionsverhältnisse“ (9) verlaufen und als „Prozess wechselseitiger Relationierungen“ (10) zu verstehen sind. ,Original" und ,Kopie" stehen in einer Beziehung zueinander, bei der das ,Original' sowohl das vorausgehende Bezugsobjekt als auch dessen nachträgliches Ergebnis ist und über diesen Prozess erst einen autonomen Status und ein Beurteilungsrecht zugesprochen bekommt:

Denn obwohl der Gegenstand, auf den im Akt des Zitierens, Kopierens, Kommentierens, Samplens etc. Bezug genommen wird, erst durch den Transkriptionsakt als zugrundeliegendes Original ausgewiesen wird, lässt ihn dieser neu erworbene Status zugleich zur Beurteilungsgrundlage für die Angemessenheit, Legitimität und Authentizität der vollzogenen Transkription werden. (10)

Es verwundert daher nicht, dass unter diesen Praktiken immer wieder Prototypen, Klassiker und Originale als vermeintlich ,primäres‘ Material gehandelt werden. Im Fall von Psycho wird dieser Status durch die Sequels und Nummerierungen zusätzlich verstärkt. Erreicht Psycho bereits zuvor durch Diskurse der Autorenpolitik den Status eines filmhistorischen Meisterwerks, so werden diese Umstände zusätzlich durch die Sequels, originalisiert‘. Dass die Vermarktung und späteren Diskurse daran festhalten, resultiert auch aus dem Bedürfnis, sowohl Psycho als auch die Sequels zu nobilitieren, auch wenn letztere weiterhin im Schatten des ersteren bleiben. 
Über die Verkettungen, wie sie Fehrmann et al. für intertextuelle Bezugnahmen und relationale Signifikationen beschreiben, werden auch Genrezusammenhänge anschaulich, weil sie Wiederkehrendes und Regelkonformes über die Verkettung mit vorausgehenden Mustern erst nachträglich generisch identifizierbar werden lassen. Es sind auch hier sekundäre Verfahren, die scheinbar vorausgehende Genremuster erst nachträglich als solche konstituieren. Der Umstand, dass ein Genre dem Film zwar logisch vorausgeht, aber zugleich faktisch nachfolgt, wurde zuvor bereits erläuert und als Differenzmoment herausgestellt. ${ }^{11}$ Neben der diskursiven Ebene von Genres, in der die Verfahren eingebettet sind und sich dialogisch verhalten, sind die intertextuellen Bezugnahmen selbst als wechselseitig zu verstehen. Auf der Ebene der Zeitlichkeit lösen sie eine Linearität auf, wie sie Katrin Oltmann auch als ,unfinished business“ (42-45) in Bezug auf die Interdependenzen zwischen Fortsetzungen, Remakes, Sequels und Prequels beschreibt. $^{12}$

Beschreibe ich die Beziehung zwischen Remake und Premake (Vorgängerfilm; K.K.) als Rückkopplung und versuche ich dieser komplexen Austauschbewegung mit der Lektürehaltung eines preposterous reading gerecht zu werden, das sowohl die Auswirkungen des früheren Textes auf den späteren als auch die Rückwirkung des Remakes auf sein Premake in die Betrachtung einbezieht, so bezeichne ich jene Effekte, die zwischen Remake und Premake durch dieses Interdependenzgeflecht freigesetzt werden, als unfinished business. (43; Herv. i. O.)

Neben der industriellen Ebene, auf der in einem ökonomischen Sinn auf Projekte, an denen Studios bereits Rechte haben, zurückgegriffen wird, interessiert Oltmann vor allem die kulturelle Ebene, auf denen Fortsetzungen arbeiten. ${ }^{13}$ Oltmann identifiziert zwischen den Versionen Rückkopplungen, aber auch Reminiszenzen (42), denen eine entscheidende kulturelle Bedeutung dahingehend zukommt, gesellschaftliche Themen - wie Fragen der Differenz und des monströsen Anderen im Horrorfilm - wiederholt zur Disposition zu stellen. Diese

\footnotetext{
${ }^{11}$ Zum Differenzmoment siehe Abschn. 2.2.4.

${ }^{12}$ Auch wenn sich die folgenden Ausführungen von Oltmann vor allem auf das Remake beziehen, können diese ebenso auf andere serielle Formen des Films erweitert werden. Die Grenzen zwischen Neuverfilmung, Sequels, Prequels, Filmserien oder auch Hommagen verlaufen auch für Oltmann fließend (20-26).

${ }^{13}$ Dieser Ebene wirft Oltmann noch eine unzureichende kultur- und medienwissenschaftliche Auseinandersetzung vor. Dieses Forschungsdefizit ist in den letzten Jahren aber zunehmend minimiert worden, wie Publikationen zu populärer Serialität zeigen (siehe u. a. Blanchet; Henderson; Kelleter, „Populäre Serialität“ und „Media of Serial Narrative“; Klein/Palmer; Loock; Loock/Verevis; Verevis, „Film Remakes“).
} 
Rückkopplungsprozesse zwischen den Produktionen beschreibt sie im Anschluss an Freuds Konzept der konstitutiven Nachträglichkeit, das aufzeigt, wie zeitlich früher stattfindende Ereignisse erst durch spätere konstruiert und erklärt werden. Für Oltmann lösen sich damit die linearen Verhältnisse auf: „Das Remake als das zeitlich Spätere ist zugleich das Frühere, weil es den ersten Film als ,Original ${ }^{*}$ erst nach dem Remaking gibt“ (29; Herv. i. O.). Auch die normative Setzung aus "qualitativ hochwertigem Original zu qualitativ minderwertigem Remake“ (ebd.) löst Oltmann in ein zirkuläres Konzept auf. Das unfinished business von Remakes gilt auch für genrespezifische Bezugnahmen und deren intertextuelle Verkettungen, da in diesen ebenso auf Früheres aus einem späteren Gestus heraus zugegriffen und erst in diesem späteren Zugriff nachträglich als generisch konstruiert wird.

Oltmanns Ausführungen sowie die Überlegungen von Kelleter und Fehrmann et al. sind für die Psycho-Fortsetzungen und deren generisches Zusammenwirken im Horrorkontext überaus produktiv anzuwenden. Sie liefern einen theoretischen Zugang zu dem Verhältnis zwischen Fortsetzungsproduktionen, in dem sie mit einem weit gefassten, poststrukturalistischen Intertextualitätsbegriff die wechselseitigen Abhängigkeitsverhältnisse verdeutlichen. Sie korrigieren die im filmhistorischen Kanondiskurs häufig ausgemacht ,Originalität" und demonstrieren die konstitutive Leistung von Sequels. Mithilfe dieser Ansätze wird im vorliegenden Fall die prominentere Position von Psycho gegenüber den Fortsetzungen erklärbar. Haben die geschickte (Selbst-)Vermarktung von Hitchcock und der Autorendiskurs Psycho zuvor im Kanon der Filmkunst etabliert, avanciert Psycho durch die Fortsetzungen zu einer diskursiven Größe, von der Anthony Perkins häufig als „Psycho One“ in Interviews spricht, und zu einem genreikonischen ,Original“, das im Horrordiskurs als „ancestor of the slasher film“ (Clover, „Men, Women“ 23) gehandelt wird. Im Kanondiskurs der Filmkunst leisten die Fortsetzungen dem vermeintlichen Original Beihilfe zu seiner Originalität, die ihnen nur eine ,Nebenrolle' gewährt und sie gar zur Unsichtbarkeit verdammt, weil sich ihre wechselseitigen Wirkungen und ihre eigene konstitutive Arbeit in der sekundären Titulierung maskieren. Diese Verhältnisse zu enthierarchisieren, beginnt damit, die „Originaltreue und die Wertigkeit der Kopie in Frage zu stellen“ (3), wie Wünsch in vielen postmodernen Adaptionstheorien beobachtet. Die Dekonstruktion des ,Originals' beginnt bereits mit der Kontextualisierung der damaligen Genrediskurse und -praktiken von Psycho (Kap. 3) und bewirkt über eine kritische Analyse der Sequels eine Aufwertung der Fortsetzungen. Auch wenn der Originalstatus als diskursive Kategorie besonders häufig in film- wie genrehistorischen Diskursen Verwendung findet, so erfolgt dies 
aus Kanonisierungsbestrebungen, Nobilitierungsvorgängen und Vermarktungszwecken heraus und nicht mit Blick auf die medialen Konstruktionsleistungen und wechselseitigen Veränderungsprozesse. Die kritische Befragung des Verhältnisses von Original und Nachfolge führt zu der Erkenntnis, dass das vermeintlich Sekundäre erst verdeutlicht, dass das ,Original' nicht so sehr ein primäres, abgeschlossenes Einzelwerk als vielmehr Teil einer offenen und kontextabhängigen Intertextualität ist, die es in ein populäres (Genre-)Feld serieller Interdependenzen und generischer Verkettungen auflöst. Anders ausgedrückt zeigt sich in diesem generisch-seriellen Umfeld, wie die Psycho-Fortsetzungen nicht nur vorab Geschlossenes öffnen und fortsetzen, sondern dieses mit aktuellen Genrekonventionen des Slasherfilms konfrontieren und so an ihrer eigenen (Genre-) Historie mitschreiben. Damit lösen sich Singularität und Originalität in eine offene Intertextualität und in fortwährende Wandlungsprozesse auf.

Dies schließt an Gemeinsamkeiten zwischen Sequels und Genre an, die filmhistorisch bis in das Kino der Attraktionen und Sensationen zurückgehen (Morsch 9) und von einer ähnlichen intertextuellen Basis ausgehen. Oltmann ersetzt den Begriff der Originalität durch Intertextualität (31), womit sie sich von einem genealogischen Ursprungskern abwendet und mit Genettes „Palimpsesten“ ein transformatives Intertextualitätsverständnis anwendet, das den filmischen „Premaketext" umformt (33). Verevis beschreibt „remaking“ als eine breit und offen angelegte Intertextualität, die als spezifische industrielle Filmpraxis verschiedene Formen der Wiederholung institutionalisiert und sich nicht auf Sequels im engeren Sinn beschränkt, wie Verevis in Bezug auf die Psycho-Fortsetzungen betont:

Understood in this way, remaking might refer to any number of cultural and industrial activities, ranging from practices of allusion and quotation, to the repetition effects which characterize the Hollywood genre film, to the cinema's ability to repeat and replay the same film over and again [sic!]. („For Ever Hitchcock“ 15).

Im Muster der Wiederholung, des Wiederkehrenden und Regelkonformen, verfügen Genres zwar über eine ähnliche serielle Praxis wie Sequels, aber erst die Einbindung kultureller Konventionen, die über Genremarkierungen hergestellt werden, leistet die entscheidende semantische Arbeit innerhalb des unfinished business. ${ }^{14}$ Es verwundert daher nicht, dass die meisten populären Sequel-Reihen im Kontext großer Genres wie dem Science-Fiction-Film oder Abenteuerfilm

\footnotetext{
14 Oltmann geht dem unfinished business in den Screwball-Komödien Hollywoods und den darin verhandelten Gender-Diskursen nach.
} 
erscheinen oder sich besonders aus dem Horrorgenre bedienen (Wünsch 8). Diesem letzteren Genre attestiert auch Clover eine Grundtendenz zur Imitation, weil die Subgenres des Horrors nicht nur ähnliche Handlungsabläufe aufweisen, sondern eben auch zahlreiche Sequels und Remakes: „Horror movies tend to be made less on the basis of audience statistics than on the basis of hunch, imitation (hence the proliferation of sequels and rip-offs).“ (,Men, Women“ 6) Clover betrachtet den Horrorfilm daher als eine Form der Folklore und der Fortsetzung mündlich tradierter Geschichten, die mit einer grundlegenden Referentialität operieren. Genres sind nach Clover ebenfalls als offene Intertexte ohne Original und ohne festen Text zu konzeptualisieren, was sie in ihrer Untersuchung der Subgenres der Slasher-, Rape-Revenge- und Occult-Filme insbesondere für das Horrorgenre geltend macht. ${ }^{15}$

This is a field in which there is in some sense no original, no real or right text, but only variations; a world in which, therefore, the meaning of the individual example lies outside itself. The ,art " of the horror film, like the ,art" of pornography, is to a very large extent the art of rendition or performance, and it is understood as such by the competent audience. (,Men, Women“ 11)

In der steten Wiederaufnahme und Variation von Themen, Motiven, Figuren und Szenen bilden Genres eigene Intertexte, die sich immer wieder selbst, aus ständiger Fortsetzung heraus, neu generieren und keinen Anfang mehr aufweisen. Demnach können sie auch kein Original mehr vorweisen, das losgelöst von der Fortsetzungsstruktur existieren kann. Dass Psycho dennoch als solches gehandelt wird, ist auf eine diskursive, keine intertextuelle Konstruktionsleistung zurückzuführen. Innerhalb der generischen Intertextualität bieten Sequels eine besondere intertextuelle Verdichtung an, die Iterationsmuster engziehen, um eine innere Kohärenz herzustellen. Solche engen Muster lassen sich in den Fortsetzungen vor allem entlang der Figur des Norman Bates, den Handlungsorten des viktorianischen Wohnhauses auf dem Hügel und des abseitig gelegenen, heruntergekommenen Motels an der Straße, der mörderischen Identität von „Mother“

${ }^{15}$ Aufgrund dieses intertextuellen Verständnisses von Genres und insbesondere des Horrorgenres als stete Variationen und schematisch-repetitive Formen wird Clovers Ansatz zur Betrachtung der ,neuen“ Tötungsszenen in den Sequels, die in einem genrespezifischen Bezug zur Duschszene als ,historischem‘ Duschmord stehen, herangezogen. Clover untersucht unter anderem archetypische Figuren und Gegenstände wie psychotische Mörder, weibliche Opfer, das Haus als Handlungsort, Waffen, Schock und Schreie sowie das Final Girl, womit sie einen geeigneten theoretischen Ansatz bietet, der nicht nur als Einzelgenretheorie auf der Ebene des Genrekonzepts greift, sondern für die Analyse genretypische Bezugspunkte bereithält. 
als Stimmkörper wie Mumienerscheinung sowie den Dusch- und Tötungsszenen beobachten. Hierbei wird von einer breit gefassten Intertextualität ausgegangen, die nicht nur zwischen Psycho und den jeweils einzelnen Fortsetzungen Psycho II, III und IV (sowie der Prequel-Serie Bates Motel) verläuft, sondern alle Produktionen in ein intertextuelles Gesamtnetzwerk einbettet und über wechselseitige Bezüge generisch-serielle Signifikationen erzeugt. In den folgenden Analysen werden die konkreten Bezugnahmen zwischen Vorgänger- und Nachfolgeproduktionen untersucht, um die spezifische Qualität der Interdependenzen - auf der Ebene des Genrekonzepts - herauszuarbeiten, die durch die wechselseitige Konfrontation von bekannten Figuren, Räumen, Szenen und ihren Genrekonventionen mit neuen Elementen und aktuellen Genrekontexten entstehen. Jede neue Fortsetzung führt die vorherigen Produktionen der Psycho-Reihe mit und bietet diese zur (erneuten) nachträglichen Relektüre an. Umgekehrt beeinflussen die vorherigen Produktionen stets auch die Wahrnehmung der nachfolgenden Produktionen und unterziehen diese einer vergleichenden Lektüre. Im Fall der Psycho-Sequels sind diese ,Re-Lektüren' äußerlich durch die fortlaufenden Titelnummern und Vermarktungspraktiken markiert, aber zugleich in den Filmen durch die Fortführung der Figuren und Orte und die Integration der Duschszene als konkrete Lesart verankert. Norman Bates und sein mörderisches Alter Ego „Mother" sowie Haus und Motel bilden übergreifende Formen der Wiederkehr, die eine ästhetische Konstanz suggerieren, aber ästhetischen Variationen unterliegen. ${ }^{16}$ Entgegen der Fortläufigkeit der Figuren und Orte wird die Duschszene aus Psycho als Zitat eingebunden und variierten Versionen wie auch neuen Tötungsszenen gegenübergestellt, sodass die Duschszene in ihrer , historischen` Prägung verstärkt wird und die neuen Tötungsszenen in ihrer genrespezifischen Aktualität hervortreten. Inwiefern diese Formen in ihrer ästhetischen und semantischen Ausgestaltung an generisch-serieller Signifikanz gewinnen und sich von Psycho lösen, wird in den Analysen aufgezeigt.

\footnotetext{
${ }^{16}$ Im weiteren Verlauf wird der kursive Sprachgebrauch von Haus und Motel verwendet, wenn die szenenbildnerische Spezifik des Wohnhauses in seiner viktorianischen Architektur und erhöhten Stellung auf dem Hügel gemeint ist und auf das Motel in seinem etwas schäbigen Design und abgelegenen Setting Bezug genommen wird.
} 


\subsection{Die Rückkehr von Figuren und Orten}

In Horror-Sequels kehren altbekannte Figuren zur Freude der Zuschauenden und ihrer Angstlust zurück, um ihre Opfer und Orte erneut aufzusuchen. ${ }^{17}$ Diese strategische Wiederaufnahme und Fortführung folgt dem generisch-seriellen Kalkül, an vertraute Geschichten und erfolgreiche Schockeffekte anzuschließen, um eine Antizipation des Schreckens als genrespezifisches Grundmoment zu erreichen. In der Fortführung von Hitchcocks Klassiker, dem ,ancestor“ des Slasherfilms, spezifiziert sich das Wissen über die Identität des Serienmörders und steigert so die Vorfreude darauf, einen solchen Schrecken neu zu erleben. In den Sequels von Psycho II-IV kehrt daher nicht nur Norman Bates zurück, sondern auch sein mörderisches Alter Ego „Mother“, das erneut von den Toten wiederaufersteht, um in Haus und Motel serienweise zu morden. Die Ironie der Horror-Sequels, dass Serienmorde am besten in Serie erzählt werden, trifft nun auch Psycho. Neben der Figur und seiner mörderischen Identität werden in den Psycho-Sequels auch die bekannten Orte reinszeniert. Diese Iteration von Normans räumlicher Rückkehr ist besonders persistent, dass Norman auch in Psycho IV, obwohl er ein neues Leben angefangen und ein neues Haus bezogen hat, wieder an diesen Ort zurückkehrt. Inwiefern die Figuren und Orte in den Sequels intertextuell variiert werden, wird zuerst an der Wiederauferstehung von Normans toter Mutter als Stimmkörper und Mumienerscheinung „Mother“ beleuchtet, die in ihrer destruktiv-monströsen Dominanz das semantische Erklärungsmuster aus Psycho nicht nur fortführt, sondern sogar noch verstärkt. Anschließend wird aufgezeigt, wie Normans Rückkehr auch Haus und Motel als Orte des Schreckens wiederbelebt und diese gerade durch ihre semantischen Variationen zu dynamischen Merkmalen des Horrors gerinnen. Dagegen markiert Anthony Perkins' kontinuierliche Verkörperung von Norman Bates eine spezifische Zeitlichkeit, an der die ,Alterung ' der Geschichte sichtbar wird und eine semantische Konstanz fortgeführt wird, die den unausweichlichen Rückfall in seine serienmörderische Identität weiterhin an ödipale Erklärungsmuster bindet.

\subsubsection{Die Wiederauferstehung von "Mother"}

Als Stimmkörper und Mumienerscheinung demonstriert „Mother“ am Ende von Psycho nicht nur ihre machtvolle Potenz über Norman, von dem sie ganz

\footnotetext{
${ }^{17}$ Der hier gewählte Sprachgebrauch von ,Zuschauenden ‘ markiert eine geschlechtsneutrale Analysekategorie jenseits heteronormativer Inskriptionen.
} 
Besitz ergriffen hat, sondern ihre psychopathologische Erscheinung wirkt in einer unheimlich-monströsen Semantik auch auf die Zuschauenden nach. Dabei entlässt Psycho mit der Gewissheit, dass mit einer Rückkehr des Untergründigen, wie der latenten Gefahr mütterlicher Dominanz, potentiell zu rechnen ist. In den Sequels hallt diese Semantik nach und unterläuft die Wahrnehmung von Normans psychopathologischer Genesung und Rückkehr. Schließlich gelingt „Mother“ die erneute Wiederauferstehung, indem sie am Ende von Psycho II in Ms. Spool, der Schwester Normans (früherer) Mutter, eine neue Mutterfigur erhält. Während sich Norman in Psycho II vor dem früheren mütterlichen Einfluss und der psychopathologischen Regression fürchtet, unterliegt er ihr in Psycho III wieder vollständig und in deutlich monströserem Ausmaß. In Psycho III wird die , neue‘ Mutterfigur zwar wieder aufgelöst, nicht ohne aber eine weitere psychotisch-mörderische Rahmung einzuziehen: Normans ,neue“ Mutter war nicht nur psychisch gestört, sondern sie hat auch seinen Vater aus Eifersucht auf ihre Schwester getötet und ihn als kleinen Jungen entführt. Darin verkehrt Psycho III das bekannte Motiv in einen destruktiv-monströsen Kindeswunsch, um Normans mörderische Psychose zu erklären.

Während Norman in Psycho II als geheilt zurückkehrt, künden zuerst nur kleine Notizen, die Norman im Haus und Diner findet, sowie ominöse Anrufe und Erscheinungen von „Mothers“ erneuter Existenz und Normans psychopathologischer Regression (Abb. 4.1a). Als erste Morde stattfinden, liegt die Vermutung nah, dass Norman als „Mother“ wieder mordet. Die silhouettenhafte und gesichtslose Erscheinung greift die Darstellungsweise aus Psycho auf, erzeugt aber keine Alptraum-Vision mehr. Denn dass weder Motel noch Haus im Psycho-Narrativ sichere Orte darstellen, gehört längst zum Vorwissen, das kleinste Geräusche wie Details von Schuhen oder Kleidersäumen und eine aufblitzende Messerklinge als drohende Gefahr markiert. Im Anblick der schwarzen Gestalt steht den Zuschauenden vielmehr die eigene generisch-serielle Vision gegenüber (Abb. 4.1b). Dennoch bleibt die wahre Täterschaft der Morde lange unklar. Den Hinweisen und Morden werden in Psycho II die verdächtige Rückkehr von Lila und die aufklärerischen Bemühungen des Psychiaters gegenübergestellt. Das ergibt ein generisches Deutungsspiel dahingehend, ob Norman wirklich wieder als „Mother" auferstanden ist. In der typischen Aufklärungsszene am Ende darf dieses Mal der Sheriff von den Intrigen und (vermutlichen) Taten Lilas und ihrer Tochter Mary berichten. Doch auch dies bleibt, wie einst in Psycho, auch in Psycho II nicht unkommentiert. Stattdessen führt Psycho II mit Ms. Spool eine neue Mutterfigur ein, die sich als die wahre Täterin herausstellt und von Norman prompt erschlagen wird. In einem Top Shot wird den Zuschauenden der Muttermord und die Wiederauferstehung von ,Mother“ als machtvoller Stimmkörper vorgeführt: „Put me in my chair. [...] Now turn me to the window. I want to keep my eyes on 
you.“ Aus diesem erhöhten Blick in die vertikale Tiefe des Raums wird die Szenerie erneut zur Fläche, zu Žižeks ,geometrische(m) Grundriss“ (213), aber statt eines Wahrnehmungsschocks birgt die Einstellung eine spielerische Verfremdung (Abb. 4.1c und 4.1d). Wie die Schachfigur eines übergeordneten Spiels folgt Norman damit nicht mehr den singulären Regeln von Psycho, sondern den seriellen Prinzipien des Sequels und Genres.

As the ending of Psycho II lays the groundwork for another sequel, it paves the way for Norman Bates' becoming a serial character that could no longer be controlled or contained by one director (Hitchcock), let alone one movie (the original Psycho). (Loock 87)
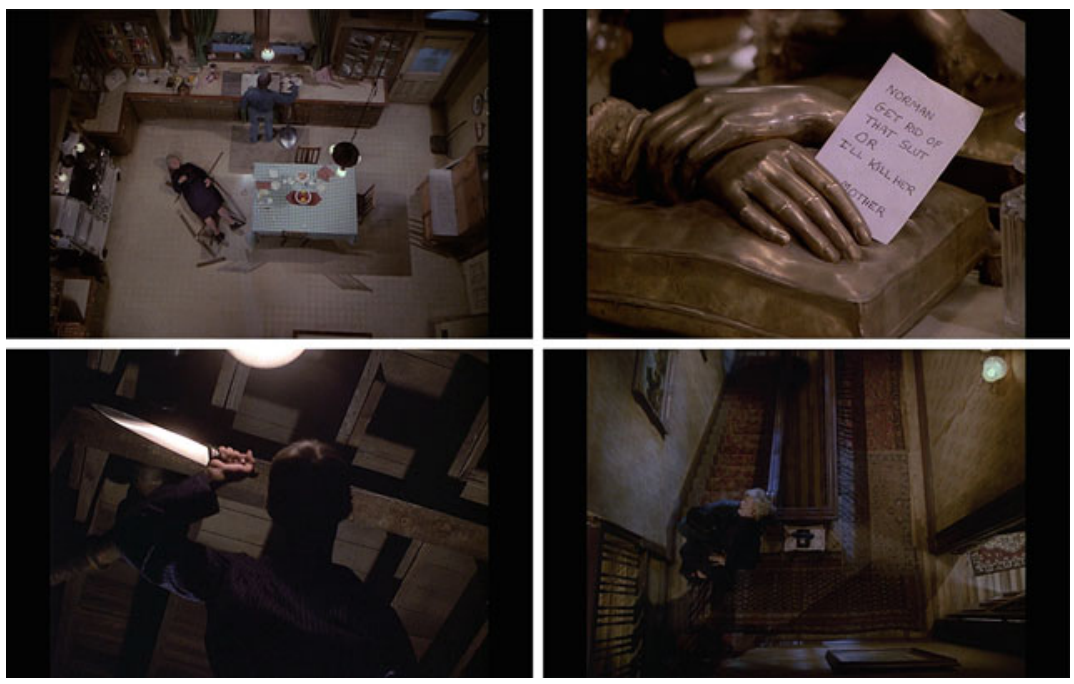

Abb.4.1a-d Psycho II, „Die Rückkehr von Mother“, USA 1983

Nach diesem Twist Ending herrscht in Psycho III erzählerische Klarheit darüber, dass „Mother“ wieder Besitz von Norman ergriffen hat und erneut mordet. In einem Flashback erinnert Psycho III an diese Szene und vergewissert die Zuschauenden der neuen generisch-seriellen Zusammenhänge und der Wiederauferstehung. Daran anschließend entwickeln sich in Psycho III die Streitgespräche zwischen Norman und „Mother“ zu einem integralen Bestandteil der Handlung. 
„Mothers“ erste Worte in Psycho III führen nicht nur die vorherigen aus Psycho II fort: „Put me closer to the window!“ Sie bedienen sich auch der früheren Anweisungen und Ausdrücke wie aus einem rhetorischen Setzkasten. Die wüsten Beschimpfungen aus Psycho stellen nun keine leeren Drohungen mehr da, sondern schaffen den Erklärungsrahmen für Normans mörderischen Wahnsinn:

„Mother": Another of your cheap, erotic delusions out of your cheap, erotic imagination. You killed her. The slut deserved it. But she's dead. And the dead don't come back. [...] You can't get rid of me. I'll always be with you, Norman. Always. Stand up straight and wipe your snotty little nose. If the disgusting little whore is going to upset you so much, just get rid of her."

In den Sequels existiert Normans mörderisches Alter Ego aber nicht mehr nur in dem Stimmkörper, sondern auch in mehreren mumifizierten Körpervariationen (Abb. 4.2a und 4.2b). Deren Anblick bewirkt aber weder eine erkenntnisvolle Demaskierung noch eine schockierende Entdeckung (,gender-shock“) wie in Psycho, sondern vielmehr geht mit den Leichengesichtern eine visuelle Variation ihrer Wiederauferstehung einher. ${ }^{18}$,Mother“ bildet in ihrer destruktiv-monströsen Dominanz als Stimmkörper und in ihrer mumifizierten Erscheinung eine wiederkehrende Horrorattraktion der Sequels, die nicht mehr nur in Psycho existiert, sondern in den Sequels eine wiederkehrende mörderische Prägnanz angenommen hat. Welchen Einfluss darauf die wiederkehrende Verkörperung durch Anthony Perkins und die unveränderte Architektur von Haus und Motel haben, wird im Folgenden beleuchtet.
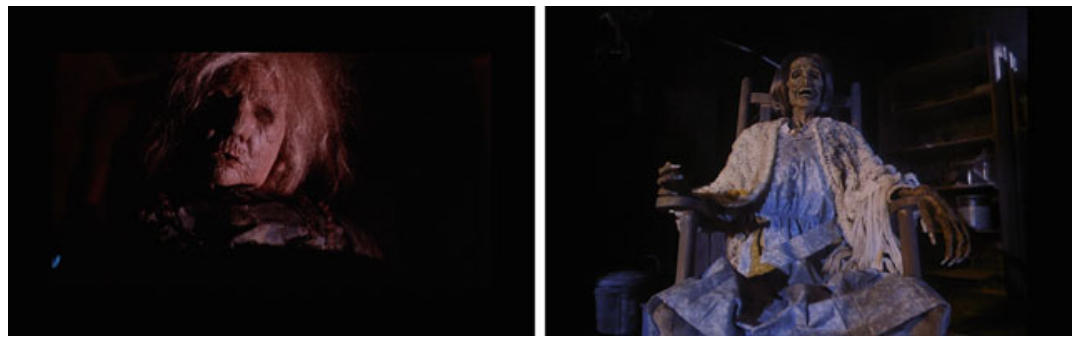

Abb. 4.2a und b Psycho III und Psycho IV, ,Der mumifizierte Leichenkörper“, USA 1986 und 1990

${ }^{18}$ Zum „Gender-Shock“ in Psycho siehe auch Abschn. 3.4.2. 


\subsubsection{Norman Bates und die Wiederbelebung der Orte}

Die wiederkehrende Verkörperung durch Anthony Perkins und die unveränderte Architektur der Orte bilden für Kelleter ,zentrale ästhetische Akteur(e)“, bei denen es ,um die Rückkehr an einen bekannten (medialen) Ort und die Wiederbelebung von Räumen (geht), durch die sich menschliche Charaktere nur wie Durchgangsreisende oder flüchtige Geister bewegen“ (,Remake“ 166). Die Figuren und Orte kennzeichnet ein relationales Verhältnis, in dem die Wiederbelebung von Haus und Motel immer wieder vertraute Szenen aufruft (wie das Schlafzimmer der Mutter in der oberen Etage), aber in der Wiederholung umkehrt: Während Norman in Psycho seine Mutter in den Keller bringt, trägt er sie in Psycho II hinauf, bevor sie in Psycho III erneut das Schlafzimmer verlässt, aber dieses Mal im Motel versteckt gehalten wird. Eine ähnliche Variation ereilt Lila, die in Psycho im Keller die mumifizierte Leiche der Mutter entdeckt und nur knapp „Mothers“ Angriff entgeht (Psycho), hingegen in Psycho II auf ironisch-tragische Weise von der neuen Mutterfigur genau dort getötet wird. Die szenische Umsetzung variiert zwar bekannte Abläufe, die Orte scheinen aber in den Sequels architektonisch unverändert. Nur von Gebüsch und einigen Bäumen umgeben, thront das Haus weiterhin kontextlos in einer kargen Landschaft über dem Motel. Sowohl die Landschaft als auch die Straße vor dem Motel führen in ein räumliches Nirgendwo. Wie die ausgeblichene Holzfassade vom Haus deuten auch der staubige Vorplatz und die schmucklose Ausstattung des Motels auf Spuren vergangener Zeiten. Abgeschieden von Highway und Sozialisation wirken die Orte merkwürdig dem Raum und der Zeit entrückt. Die viktorianische Architektur erinnert zwar an Motive des gothic castle, und in den nächtlichen Inszenierungen an düstere Erzählungen der gothic novel, deren ,alte` Horrorgeschichten Monster aus weiter Ferne bringen (Podrez, „Der Horrorfilm“ 7), aber der Ort ist als haunted house in einen modernen Horror übergegangen, der Angst und Schrecken statt aus weiter Ferne in einer unmittelbaren Nähe und Alltäglichkeit erzeugt (Tudor, „Monsters“ 190). ${ }^{19}$ Auch der zwielichtige Ruf von Motels, die der FBI-Direktor J. Edgar Hoover einst als „Camps of Crime“ bezeichnete und die in pulp magazines des True Crime-Genres wie billigen Soft-Porn-Taschenbüchern noch bis Mitte der 1960er eine ganze Reihe skandalöser Motelgeschichten hervorbrachten, ist

${ }^{19}$ Zum haunted house siehe auch Podrez („Unheimlich lebendig“). 
längst hinter einer neuen Medialisierung von Sexualität und Verbrechen zurückgefallen (Henry). ${ }^{20}$ Vor diesem genre- und kulturhistorischen Hintergrund sind Haus und Motel von ihrer eigenen kulturellen Semantik, die in Psycho noch präsent war, überholt worden. Dennoch werden Haus und Motel in den Sequels als Orte des Schreckens wiederbelebt. Erscheint das Motel in Psycho zuerst noch als surrealer Ort der Beruhigung, entpuppt es sich mit der Duschszene allerdings als eine Alptraum-Vision, auf die im Haus weitere Todes-Erscheinungen folgen. Während das Haus in Normans 23-jähriger Abwesenheit nur etwas eingestaubt wirkt (Abb. 4.3a), ist das Motel in Psycho II derweil zu einem dubiosen Stundenmotel heruntergekommen (Abb. 4.3b). Mit Normans Rückkehr in Psycho II distanzieren sich die Sequels zugleich von ihren vorherigen Erscheinungen, was bereits der Umstand zeigt, dass die Orte nicht nur neu bezogen, sondern auch renoviert werden: So streicht Norman in Psycho II die Fassade des Motels und unterstreicht damit buchstäblich den Wunsch nach einem Neubeginn, der zudem nun ,in Farbe ' erfolgt (Abb. 4.3c). ${ }^{21}$ Mit Normans Wiedereinzug gerät das Haus in Psycho II zum surrealen Schauplatz einer Reihe unerklärlicher Erscheinungen und neuer Morde (Abb. 4.3d).

\footnotetext{
${ }^{20}$ In den 1920er Jahren entstanden Motels als funktionale und billige Unterkünfte an den Highways und Reiserouten und bildeten kulturelle Symbolträger für eine mobile Moderne und einen neuen Individualismus in Amerika. Zwar lassen sich historisch kriminelle Einzelfälle ausmachen, in denen Gangster Motels als Verstecke nutzten und sich Schießereien mit der Polizei lieferten. Die vermeintliche Gefahr dieser ,hotbeds of sordidness and immorality“ und „Camps of Crime“ ist allerdings reine Fiktion. Mit dem Anstieg nationaler Motelketten stiegen auch der Komfort und die Respektabilität von Motels, was dazu führte, dass das mediale Interesse an diesen unmoralischen Orten schwand. Einen kurzen kulturgeschichtlichen Abriss gibt Henry.

${ }^{21}$ Auch in dem Pilotfilm Bates Motel von 1987 renoviert Alex, der von Norman Bates das Anwesen geerbt hat, das Motel aufwendig. Eine Kurzdarstellung des Pilotsfilms findet sich in der Einleitung.
} 

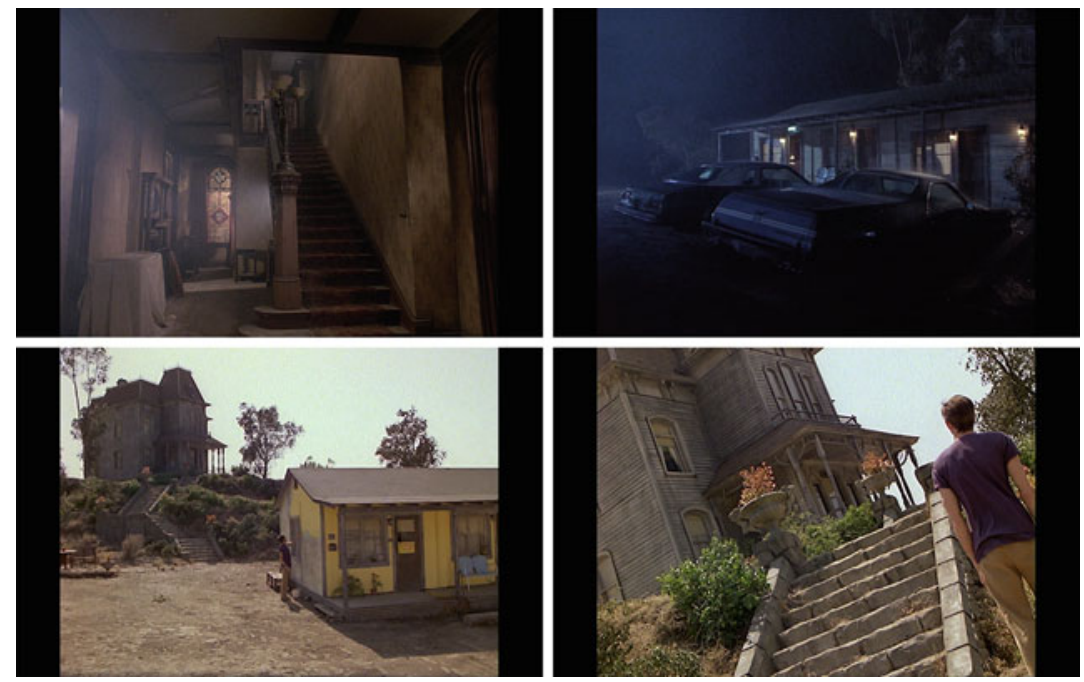

Abb.4.3a-d Psycho II, „Haus und Motel“, USA 1983

Demgegenüber bietet sich in Psycho III ein lebensferner Anblick auf Haus und Motel, die auch in der glühenden Hitze nichts von ihrer schaurigen Erscheinung eingebüßt haben (Abb. 4.4a). ${ }^{22}$ Die mit der jungen Novizin Maureen Coyle gesetzte Glaubensthematik entspinnt sich in Psycho III zu einer religiösen Symbolik, die in der Dualität zwischen Haus und Motel Ausdruck findet: Während das Motel zu einer Brutstätte sündiger und teuflischer Gestalten gerinnt (Abb. 4.4b), verwandelt sich das Haus in eine Heiligenstätte (Abb. 4.4c). ${ }^{23}$

\footnotetext{
${ }^{22}$ Seit 1974 ist mit The Texas Chain Saw Massacre solch ein vertrocknetes Setting als Ort des Schreckens für einen Horrorfilm durchaus typisch.

${ }^{23}$ In Psycho III greift die religiöse Motivik auch bei den Figuren, die als Gegensatzpaar erscheinen: Die Figur der Maureen wird trotz ihrer Glaubenszweifel in eine Symbolik des Reinen, Guten, gesetzt, während bei der Figur des Duke Motive des Sündigen und Teuflischen greifen.
} 


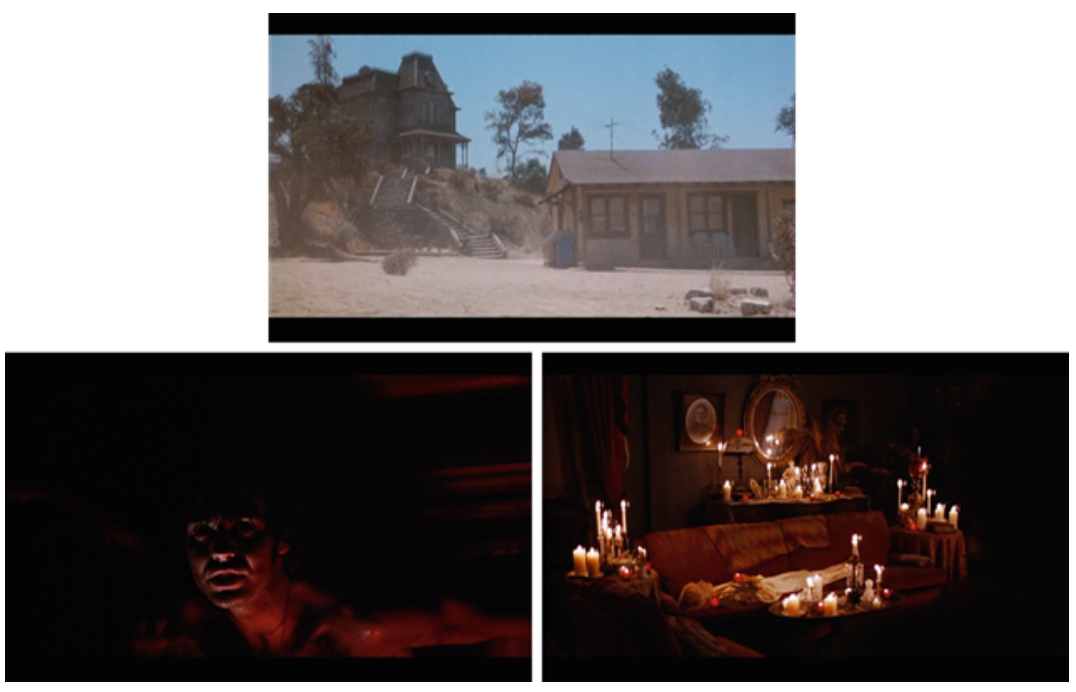

Abb.4.4a-c Psycho III, „Haus und Motel“, USA 1986

Schließlich führen die Rückblenden in Psycho IV in die Anfangsjahre zurück als auch das Haus noch in frischer Farbe glänzte (Abb. 4.5a), bevor es Norman am Ende in Brand setzt, um sich endgültig den Fesseln seiner Vergangenheit zu entledigen (Abb. 4.5b). Doch die düstere Vergangenheit des Hauses eilt nicht nur den Rückblendungen in Psycho $I V$ voraus und täuscht in dem hellen Fassadenanstrich nicht darüber hinweg, dass Norman zum Serienmörder werden wird; sie ist auch in der Gegenwartserzählung weiterhin präsent. In den Flammen erscheinen Norman schließlich die ,Geister' seiner Vergangenheiten. Auch wenn er sich aus ihren Fängen noch knapp befreien kann, hält das letzte Sequel den Ort für wiederkehrende Horrorgeschichten offen und lässt über die Ruinen „Mother“ unnachahmlich auf eine Wiederauferstehung drängen: „Let me out!“ bildet der erneute Ruf nach einer Fortsetzung. 

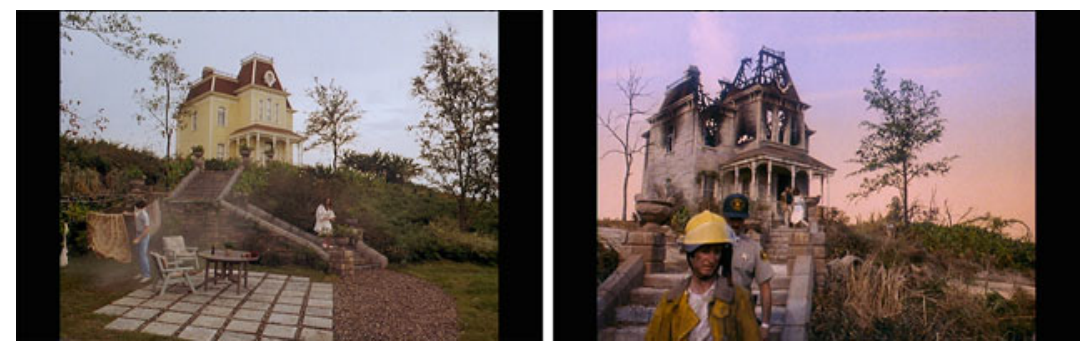

Abb. 4.5a und b Psycho IV, „Das Haus damals und heute“, USA 1990

Mit Anthony Perkins findet in den Sequels eine spezifisch sichtbare Verkörperung von Norman Bates statt, wie sie für Serienmörderfiguren innerhalb des Slashergenres sonst eher untypisch ist. Die bekanntesten unter ihnen verstecken sich hinter weißen Schimützen (Michael Meyers in der Halloween-Reihe), Hockeymasken (Jason in der Friday the 13th-Reihe) oder sind durch vernarbte Gesichter (Freddy Krueger in der The Nightmare on Elm Street-Reihe) nicht zu erkennen. Diese seriellen Genrefiguren führen ein von Schauspielerkörpern entzeitlichtes Dasein, was sie zugleich zu ewiger Wiederkehr befähigt. In der wiederkehrenden Verkörperung stellen dagegen die Psycho-Sequels eine ikonische Verkörperung zwischen Figur und Schauspieler her, die nach dem Tod von Anthony Perkins 1992 weitere Fortsetzungen vorerst verhindert. ${ }^{24}$ Bereits in seiner Kritik zu Psycho II erkennt Andrew Sarris, dass Normans Figur in ein serielles Leben übergegangen ist: „He is no longer part of Sir Alfred's personal nightmare. His Norman Bates has taken on a life of his own [...]." (,Norman“ 45 f.) In den Sequels eilen der Figur ihre psychotischen Abgründe voraus. Auch entspricht die Figur nicht mehr der jugendlichen und harmlosen Erscheinung.

Die 23 Jahre haben sich auch in Perkins Körper eingeschrieben. Die Figur zeichnet aber weiterhin die freundliche Naivität aus, die in Psycho zur Komplizenschaft verführt. Angesichts des Vorwissens und der Erwartungen an eine Fortsetzung kann sich Norman zwar der generisch-seriellen Zwangsläufigkeit nicht mehr entziehen, die ihn zum Serienmord zwingt; aber gegenüber seinen Wiederholungstaten halten die Sequels weiterhin eine Unschuldigkeit aufrecht. Wie bereits in Psycho bildet Norman das Opfer einer puritanisch-dominanten Über-Ich-Mütterlichkeit, die in den Sequels zu einem antagonistischen Verhältnis ausgebaut wird. Angesichts Normans unausweichlicher Entwicklung zum

\footnotetext{
${ }^{24}$ Der Pilotfilm Bates Motel von 1987 kann als Scheitern des Versuchs gewertet werden, die Geschichte losgelöst von Anthony Perkins und dem ursprünglichen Titel fortzuführen. Das Remake von 1998 wiederum bewegt sich außerhalb der genrespezifischen Aktualisierung, wie sie für die Fortsetzungsgeschichten gilt.
} 
erneuten Serienmörder bedienen die Sequels umso vehementer das ödipale Erklärungsmuster und führen seinen psychopathologischen Wahnsinn auf eine monströsere Mutterfigur zurück. In Psycho III besitzen ihre wüsten Beschimpfungen eine deutliche mörderische Intentionalität, die sich letztlich als psychisch gestört herausstellt und in einen monströsen Kinderwunsch verkehrt, was Psycho II einer nachträglichen Relektüre unterzieht. Die Rückblenden in Psycho $I V$, die eine erste Vorgeschichte zu Normans Genese als Serienmörder erzählen, führen zudem Normans psychopathologischen Wahnsinn auf ein inzestuöses Verhältnis zur Mutter zurück. Kurzzeitig gelingt ihm am Ende von Psycho III die Emanzipation. In seiner finalen Erscheinung als „Mother“ heftet sich „Mothers" Stimme nicht mehr fremdartig an Norman, sondern spricht in ihrer wahren Intentionalität (,Get her, boy. Get her, just like the others!“‘) direkt aus ihm heraus. Das Schockmoment und die subversive Monstrosität, die Normans Auftritt im Keller, seine unschuldige Erscheinung auf der Polizeiwache und „Mothers“ ,harmlose" Worte in Psycho noch erzeugen, verkehren sich in Psycho III in einen neuartigen direkteren Wahnsinn: Normans irres Lachen und „Mothers“ Stimme erscheinen im Kerzenlicht wie eine teuflische Inkarnation (Abb. 4.6a). Das in den Sequels generierte Mehrwissen über Normans psychotische Existenz scheint in der diesmaligen Zusammenführung von Trägerkörper und Stimme eine Deakusmatisierung nach Chion zu ermöglichen, die die mütterliche Stimme entmachtet und Norman von ihrem mörderischen Einfluss zu erlösen scheint. Trotz der Festnahme bleibt für Norman eine gelungene Subjektwerdung nicht möglich (,I'll finally be free.“), eine derartige (Auf-)Lösung ist im Sequel nicht vorgesehen. Norman bleibt auch am Ende von Psycho III weiterhin fest im mütterlichen Griff. Wenn Norman in der letzten Einstellung verstörend in die Kamera lächelt (Abb. 4.6b), bietet auch Psycho III ein offenes Ende an, das mit der generisch-seriellen Gewissheit entlässt, dass mit seiner Rückkehr jederzeit zu rechnen ist.
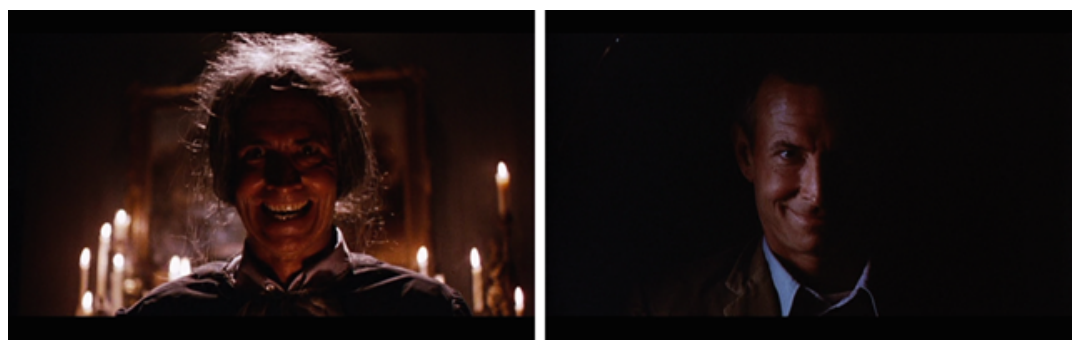

Abb.4.6a und b Psycho III, „Das Fratzengesicht“, USA 1986 
Innerhalb der Sequels nehmen die Orte und Figuren als dynamische Genremerkmale eine übergreifende generisch-serielle Signifikanz an, weil sie sowohl in ihren wiederholten Inszenierungen Bezüge zu den Vorgängerproduktionen schaffen als auch erzählerische Verläufe verkehren und spezifische Semantiken ausprägen. Während die Sequels „Mothers“ Wiederauferstehung als generischserielle Attraktion und monströse Semantik festschreiben, erfahren Haus und Motel als wiederkehrende Orte des Schreckens in ihrer Wiederbelebung semantische Variationen. Gegenüber der zeitlichen Entrücktheit dieser Orte geht von Perkins " Norman eine spezifische Zeitlichkeit aus, die sowohl eine zeitliche Fortdauer schafft, aber über den schauspielerischen Körper auch symptomatisch für eine gealterte Genregeneration von Psycho steht. Dieser werden Mary (Psycho II), Maureen (Psycho III) und weitere jüngere Figuren gegenübergestellt, die für eine neue Generation des Slasherfilms stehen und entsprechend den Darstellungskonventionen auch als Opfer getötet werden. Inwiefern diese Gegenüberstellung insbesondere entlang der Duschszenen forciert wird, wird nachfolgend betrachtet.

\subsection{Die Duschszene als ,historische' Genresignatur}

Neben den Orten und Figuren nehmen die Sequels auch an der Duschszene aus Psycho wechselseitige Relektüren vor, indem sie die Duschszene zuerst als ,primäres' Objekt herausstellen, bevor in eigenen Versionen strukturelle Abweichungen (Psycho II) und semantische Umdeutungen (Psycho III) vorgenommen werden. Die Duschszene wird hierbei als ,historische' Genresignatur festgeschrieben, deren Schrecken zwar nicht mehr aufgerufen werden kann, aber die sich dennoch auf die Wahrnehmung der Fortsetzungserzählungen und der , neuen' Duschszenen auswirkt. Zuerst wird an Psycho II aufgezeigt, wie die Duschszene als Prolog, oder auch pre-credit sequence, zum ,originären Duschmord transformiert wird und die Duschszenen der neuen Handlung über eine bedrohliche Semantik unterläuft. Anschließend wird an Psycho III deutlich, wie die Duschszene sich als abrupter Flashback einschiebt und zu einem unüberwindbaren Erinnerungsmoment erhebt, das eine Perspektivierung Maureens als Marions Doppelgängerin vornimmt und ihre Ankunft im Motel und die folgende Duschszene zwar determiniert, aber von einer Horror- in eine ,Errettungsvision “ verändert. 


\subsubsection{Der ,originäre' Duschmord in Psycho II}

Die Duschszene bildet den Auftakt der Fortsetzungsgeschichte von Psycho II, der sie als Prolog nicht nur vorangestellt wird, sondern in der sie auch Normans Rückkehr als narrative Folie begleitet. Die Szene setzt mit der Leuchttafel des ,Bates Motel ${ }^{`}$ ein, auf den Marions Gang in das Badezimmer folgt, und endet mit dem Blick durch das Motelzimmerfenster auf das Wohnhaus, wenn Normans Aufschrei erklingt: „Mother! Oh, God, Mother! Blood! Blood!“ Aus dem narrativen Kontext von Psycho herausgelöst, wird die Duschszene über zwei räumliche Bezugspunkte, Motel und Haus, eingerahmt. Das Zitat ruft aber nicht einfach den mörderischen Ablauf und die räumliche Verortung in Erinnerung; die Einbindung in Psycho II nimmt spezifische formalästhetische Veränderungen vor, die die Szene generisch-seriell neu verbinden. Zwei formale Aspekte sind hierbei markant, die allerdings nur in einem direkten Vergleich auffallen und für das Durchschnittspublikum kaum zu erkennen sind. Erstens variiert das Format zwischen der Kino- und späteren DVD-Veröffentlichung, was einen ästhetischen Bildzugewinn zur Folge hat, und zweitens ist die Szene um Einstellungen gekürzt worden. ${ }^{25}$ Gegenüber dem ursprünglichen 1.85:1 Widescreen-Kinoformat von Psycho ist das Bild in Psycho II in ein 1.33:1 Fernsehformat ,geöffnet" und erhält so einen Bildzugewinn (Abb. 4.7a und 4.7b), der für ein filmhistorisches Verständnis nicht zu unterschätzen ist (Distelmeyer 99 ff.; Heller, „Prettier than Ever"). ${ }^{26}$ Die (restaurierten Neu-)Veröffentlichungen auf DVD, Blu-Ray und Streaming-Plattformen prägen buchstäblich unser ,Bild` der Vergangenheit. Auch wenn die entscheidenden Bildinhalte horizontal ausgerichtet sind und der Bildzugewinn nicht unbedingt zusätzliche narrative Informationen liefert, schließen die zusätzlichen Details wie der Duschkopf, die Halterungsleiste und der Türrahmen vor allem den oberen Bildbereich anders ab, statt einen fließenden Übergang in

${ }^{25}$ Grundlage bildet die DVD-Edition Psycho Collection I-IV von Universal Pictures Germany aus dem Jahr 2007.

2635 mm-Negativfilm hat ein Bildformat von 1.37:1. Für das seit 1956 gängige WidescreenKinoformat wird der Film komplett belichtet, aber in der Kinoprojektion durch den Leinwand-Cache oben und unten maskiert, um ein Breitwandbild von 1.85:1 zu erhalten. Dies sollte vor allem auch Kinos mit älterer Projektionstechnik und fehlender vertikaler Maskierung helfen, die Leinwand besser auszufüllen. Für das Fernsehen wurden die Filme meist als „open matte“ unmaskiert gezeigt, womit mehr Bildmaterial im oberen und unteren Bereich sichtbar wurde. Gegenüber der Demaskierung wurde auch oft das Pan\&ScanVerfahren eingesetzt, bei dem von den ursprünglichen Weitbildformaten links und rechts abgeschnitten wurde, um das 1.33:1 Fernsehformat zu erhalten. Allerdings tritt dabei ein Bildverlust von fast einem Drittel (28\%) auf. Zur Differenz zwischen Formatversionen siehe neben Distelmeyer auch John Beltons Studie zum Widescreen. 
das Off des Bildes zu leisten. Bereits an diesen minimalen Formatänderungen zeigt sich, dass die in Psycho II vorangestellte Duschszene nicht einfach aus Psycho ,herauskopiert ' und unverändert einfügt wird, sondern einem verändernden Zugriff unterliegt, der in der formattechnischen Öffnung Psycho bereits als vorausgehendes , Original` demaskiert.
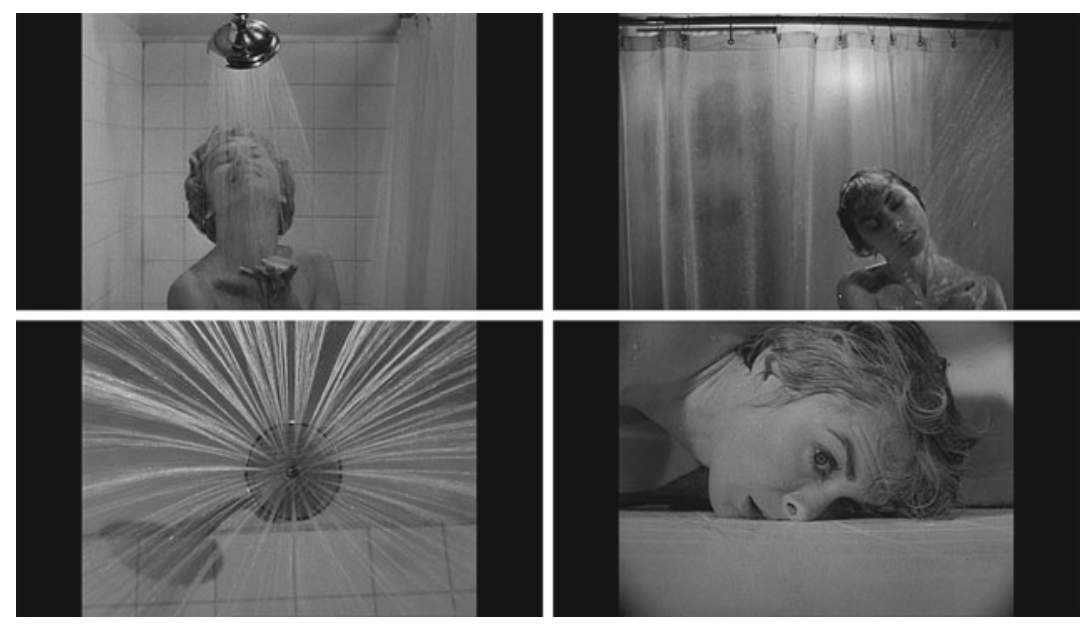

Abb.4.7a-d Psycho II, „Formatänderungen und Kürzungen der Duschszene“, USA 1983

Auffälliger noch als das Format, jedoch auch nur im direkten Vergleich erkennbar, sind die vorgenommenen Kürzungen, die in den szenischen Ablauf eingreifen. Unter anderem fehlt die Toiletten-Einstellung, in der Marion die zerrissenen Papierschnipsel hinunterspült, welche ihren Diebstahl auflisten und von Lila innerhalb der Detektivhandlung als Beweis ihres Aufenthalts im Motel gefunden werden (nicht alle Schnipsel sind in die Toilette gefallen). Des Weiteren wurde die Abfluss-Einstellung herausgeschnitten, die nach Marions Tod dem fließenden Wasser folgt und in einer soghaften Drehung in Marions totes Auge überblendet. Stattdessen betritt Marion unmittelbar die Dusche und nachdem sie tot über den Badewannenrand fällt, wird von der Frontalaufnahme des Duschkopfs direkt auf Marions leeren Blick gewechselt (Abb. 4.7c und 4.7d). Damit ist die Szene nicht nur aus ihrem äußeren Narrationskontext, sondern auch von jenen generischen Markierungen gelöst, die sie in Psycho noch mit der vorgängigen Raubgeschichte des Thrillers sowie der nachfolgenden Detektivgeschichte 
verbindet und der audiovisuellen Brutalität noch eine indifferente Subkomponente verleiht. Die Kürzungen lösen die Duschszene aus diesen generischen Kontexten heraus, sodass sie als Zitat nur noch jenes innerszenische Moment markiert, in das die Gewalt als pointierte Brutalität, als "slicing and physical penetration“ (Kendrick, „Hollywood Bloodshed“ 43), in das intim-erotische und ungeschützte Moment der Dusche Einzug hält und ihr weibliches Opfer findet. Der überraschende und destabilisierende Effekt, der in Psycho noch mit der Tötung der Hauptfigur entsteht und in einer filmischen Gleichgültigkeit mündet, die das gleichförmige Weiterrauschen des Wassers und die soghafte Wirkung der AbflussEinstellung erzeugen, werden in Psycho II nicht mehr aufgerufen. Woher Marion kommt und warum sie im Bates Motel ist, interessiert in der Fortsetzung nicht mehr.

Die Formatänderung, aber insbesondere die Kürzungen, unterstreichen, was Distelmeyer als ,anti-originale Eigenschaft des Films“ (101) beschreibt: Es existiert keine fixe Größe ,Film', „,ie an irgendeinem Ort zu einer bestimmten Zeit in einem Kino gezeigt worden ist" (102) oder im vorliegenden Fall reproduziert werden kann. ${ }^{27}$ Ein vorausgehendes Original im Sinne eines stabilen Werkzusammenhangs kann nur diskursiv suggeriert werden, wobei dieser originäre Status durch den Transfer in einen neuen narrativen Kontext zugleich unterlaufen wird. Auch in Psycho II wird die Duschszene nur diskursiv als ,originale Duschszene" gesetzt, indem sie prominent an den Anfang gestellt wird, aber damit Psycho de facto nachträglich ist. Darin folgt Psycho II dem bereits bestehenden Diskurs, der Psycho und die Duschszene längst als Klassiker und meisterliche Genreikone des Horrors kulturell genormt hat. Zugleich zeigt ein genauer Blick auf, dass diese kulturelle Normung im Material selbst nicht ohne Weiteres angelegt ist, sondern die spezifische ,Originalität ' einiger medialer Veränderungen bedarf, um als pointierte Genremarkierung des Horrors zu funktionieren. Erst die Lösung vom narrativen Kontext und den generischen Verbindungen in Psycho verändern die Szene zu dem brutalen Duschmord, als der sie im Diskurs bereits existiert und als welcher sie schließlich repräsentativ für Psycho an den Anfang der Fortsetzung gesetzt werden kann. Indem Psycho II die Duschszene an ihren diskursiven Status anpasst, greift sie retrospektiv in die Materialität von Psycho ein und führt den schon bestehenden Diskurs auf filmischer Ebene fort. Als ikonischer Duschmord konstituiert sich die Duschszene damit erst im Nachhinein.

${ }^{27}$ Insbesondere im digitalen Umfeld von DVD und Blu-Ray erlaubt die Auswahl an Format, Sprache und Kommentaren eine Reihe medienspezifischer Veränderungen (Distelmeyer 101). 
Abb.4.8 Psycho II, „,Der Blick auf das Haus", USA 1983

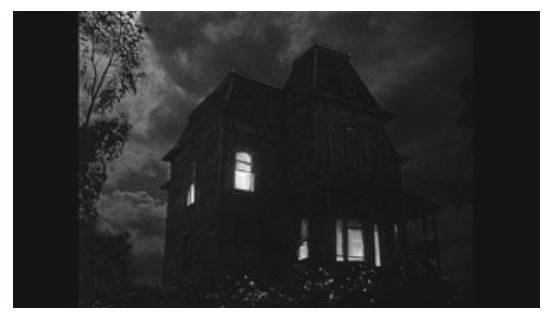

Auswirkungen auf Psycho bewirkt die Duschszene auch in Bezug auf Psycho II Rückkopplungen. Aus der letzten Haus-Einstellung schält sich die Fortsetzungshandlung heraus, wobei im genauen Vergleich zu Psycho geringe Unterschiede in der Perspektive und den Bilddetails festzustellen sind, die das Haus in seiner architektonischen Gesamtheit ohne die seitliche Gardine zeigen (Abb. 4.8). Es handelt sich in Psycho III um eine neue Aufnahme, die den Blick auf das Haus und die Schwarzweiß-Ästhetik von Psycho imitiert und sich so als ,frühere' Aufnahme kaschiert. Dabei nehmen die visuellen Veränderungen, die ebenfalls ohne direkten Vergleich nicht zu erkennen sind, bei genauer Betrachtung eine wichtige Fokusverlagerung vor, die den Blick von zusätzlichen Rahmungen befreit und das Haus in der Bildmitte zentriert, sodass sich in der anschließenden langen Einstellung der Blick auf das Haus intensivieren kann und das Haus als dunkle und düstere Erscheinung vor dem hellen Nachthimmel umso expressiver hervortreten lässt. Diese Aufnahme ist der Ausgangspunkt eines langsamen transformativen Übergangs, in dem sich Psycho und Psycho II ineinanderschieben und wechselseitig verschieben. Nach Normans Aufschrei geht die Schwarzweiß-Einstellung langsam in eine blaue Sättigung über (Abb. 4.9a), auf die mit ,Anthony Perkins “ die Cast Credits eingeblendet werden, bevor in einer Schwarzblende der Titel ,Psycho II ' folgt. ${ }^{28}$ Während sich die bereits blau gefärbte Nachtaufnahme in ein warmes Gelb der Morgendämmerung auflöst (Abb. 4.9b) und schließlich in volles Tageslicht und blauen Himmel übergeht (Abb. 4.9c), scheint nicht nur die nächtliche Düsternis von Psycho verschwunden, sondern in Psycho II scheinen auch die

${ }^{28}$ Zur Titelgestaltung, die das Design von Tony Palladino aus den Psycho-Materialien aufgreift, siehe Abschn. 3.3.3. Die Credits in Psycho II verbinden die Produktionen wechselseitig. Bereits über den letzten Einstellungen der Psycho-Duschszene werden erste Credits eingeblendet, die auf den neuen Produktionskontext verweisen. Doch erst der Hauptdarsteller ,Anthony Perkins“ zieht eine gemeinsame Produktionsreferenz ein. Während die ,alten “ Bilder bereits vorauseilend als , neue ' Produktion markiert werden, erscheint die ,neue "Aufnahme noch als Psycho. 
Jahre zwischen 1960 und 1983 nahtlos ineinander überzugehen und sinnbildlich einen Neuanfang einzuleiten.
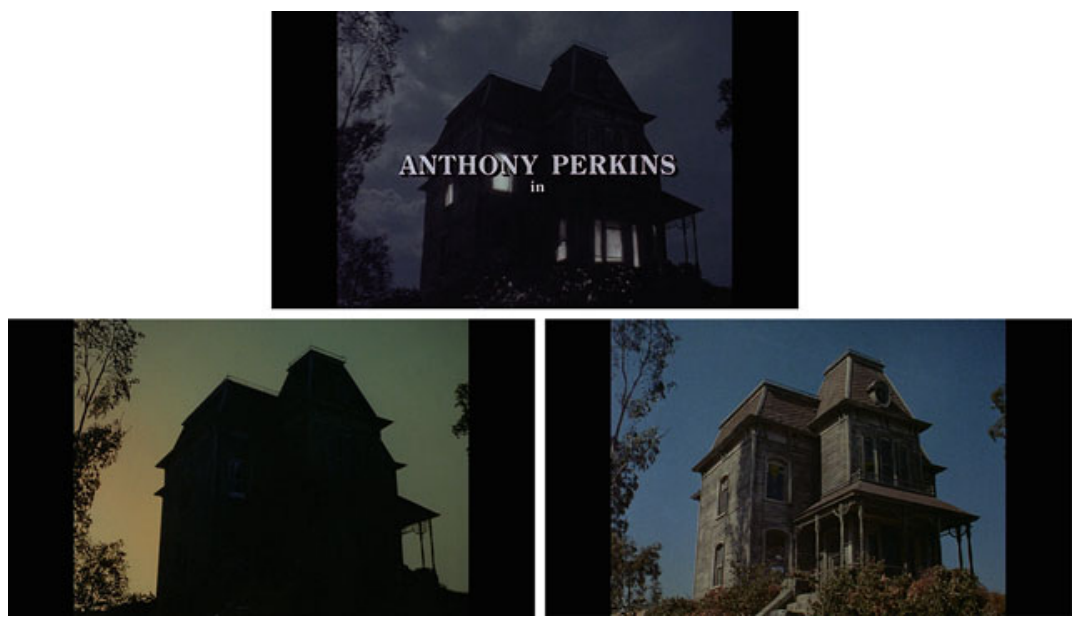

Abb.4.9a-c Psycho II, „Transformation des Hauses“, USA 1983

Über die Duschszene werden beide Produktionen wechselseitig in Bezug zueinander gesetzt und die zwischen ihnen liegenden Jahrzehnte ästhetisch überbrückt. Das schließt insofern an die vorherigen theoretischen Ausführungen zu den generisch-seriellen Interdependenzen an, als dass Psycho zwar als vorherige primäre Produktion markiert wird, sich ihr originärer Status aber erst nachträglich über die in Psycho II geleistete Kürzung und den transformativen Übergang generiert. Psycho II stellt die Duschszene als genrespezifisches Initiationsmoment der eigenen Erzählung voran, die als brutaler Duschmord nicht nur , originär ' auf $P s y$ cho zurückverweist, sondern auch die Fortführung in Psycho II als Slasherfilm vordeutet.

Dieses gezielte, wechselseitige Verbindungsverfahren erhebt die Duschszene zum vorausgehenden Bezugsobjekt, aber es generiert dieses in seiner dafür nötigen markanten Form und generischen Signifikanz erst nachträglich. Die Anbindung verläuft folglich gegenläufig: Nicht Psycho wird mit Psycho II verbunden, auch knüpft Psycho II nicht einfach an Psycho an, sondern vielmehr greift 
Psycho II transformativ auf Psycho zu. Erst darüber werden der Duschszene im Anschluss an Fehrmann et al. ein autonomer Status und ein Beurteilungsrecht für den nachfolgenden Film zugesprochen. Sie wird hierdurch zu demjenigen Standard erhoben, an dem es sich Psycho II messen lassen will. Dabei kann die Schockwirkung, die 1960 noch in der überraschenden Tötung der Hauptfigur entsteht, so nicht mehr hergestellt werden. Die Signifikanz der Duschszene ist von historischer Prägung, denn sie steht ästhetisch für ein state of art-Horrorkino von 1960, das nicht nur als Zitat funktioniert, sondern auch als Archivbild betrachtet werden kann. Mit den Überlegungen von Matthias Steinle zum Archivbild als „Zeichenkomplex“ und „Wahrnehmungsphänomen“, das sich ,aufgrund ästhetischer Markierungen zeitlich in der Vergangenheit und räumlich in einem anderen Ursprungskontext situiert“ (262 f.), ist auch die Duschszene in Psycho II als ,bereits existent " semantisch-medial präformiert und strahlt eine Autorität als authentisches Zeitzeugnis bzw. Genrezeugnis aus. Durch die Wiederverwendung wird der Szene ähnlich den Archivbildern nach Steinle besondere Signifikanz zugesprochen. Ihr Schrecken jedoch kann als genrespezifisches Grundmoment nicht mehr erlebbar gemacht werden. Im Kontext von Psycho II kommt ihr daher eine neue Funktion zu. Die Duschszene wirkt im Anschluss an Kelleter an der eigenen medialen (Selbst-)Historisierung mit. Indem sie gegenüber anderen Elementen als erinnerungswürdig herausgelöst und als signifikant vorangestellt wird, verweist sie als , historischer' Duschmord nicht nur retrospektiv auf Psycho, sondern bildet in Psycho II den Auftakt einer genrespezifischen Mordserie. Auf generisch-serieller Ebene geht damit von Psycho II die ,produktive Kraft ${ }^{\circ}$ des Genres aus, wie sie Grindon (49) und Berry-Flint (26) beschreiben, denn indem das Sequel die Duschszene verändert, öffnet es die als abgeschlossen angelegte Erzählung von Psycho nachträglich für eine Fortführung und bindet diese in einem transformativen Übergang prospektiv an eine neue Horrorerzählung. In der Verbindung bildet sie im Sinne Oltmanns ein unfinished business, das nicht nur auf den früheren Text rückwirkt, sondern sich auch auf die Wahrnehmung der , neuen' Duschszene in Psycho II auswirkt.

\subsubsection{Abweichende und bleibende Gefahren in Psycho II}

Als in Psycho II die junge Kellnerin Mary Samuels in das Haus einzieht und das Badezimmer in der oberen Etage benutzt, erfolgt eine Reinszenierung der Duschszene, die ähnlich wie in Psycho nach dem ersten Drittel des Films platziert ist und zuerst das gleiche Einstellungsarrangement bedient (Abb. 4.10a-4.10f): Die Kamera folgt ihren Füßen, wie sie die Dusche besteigt und den Vorhang schließt, 

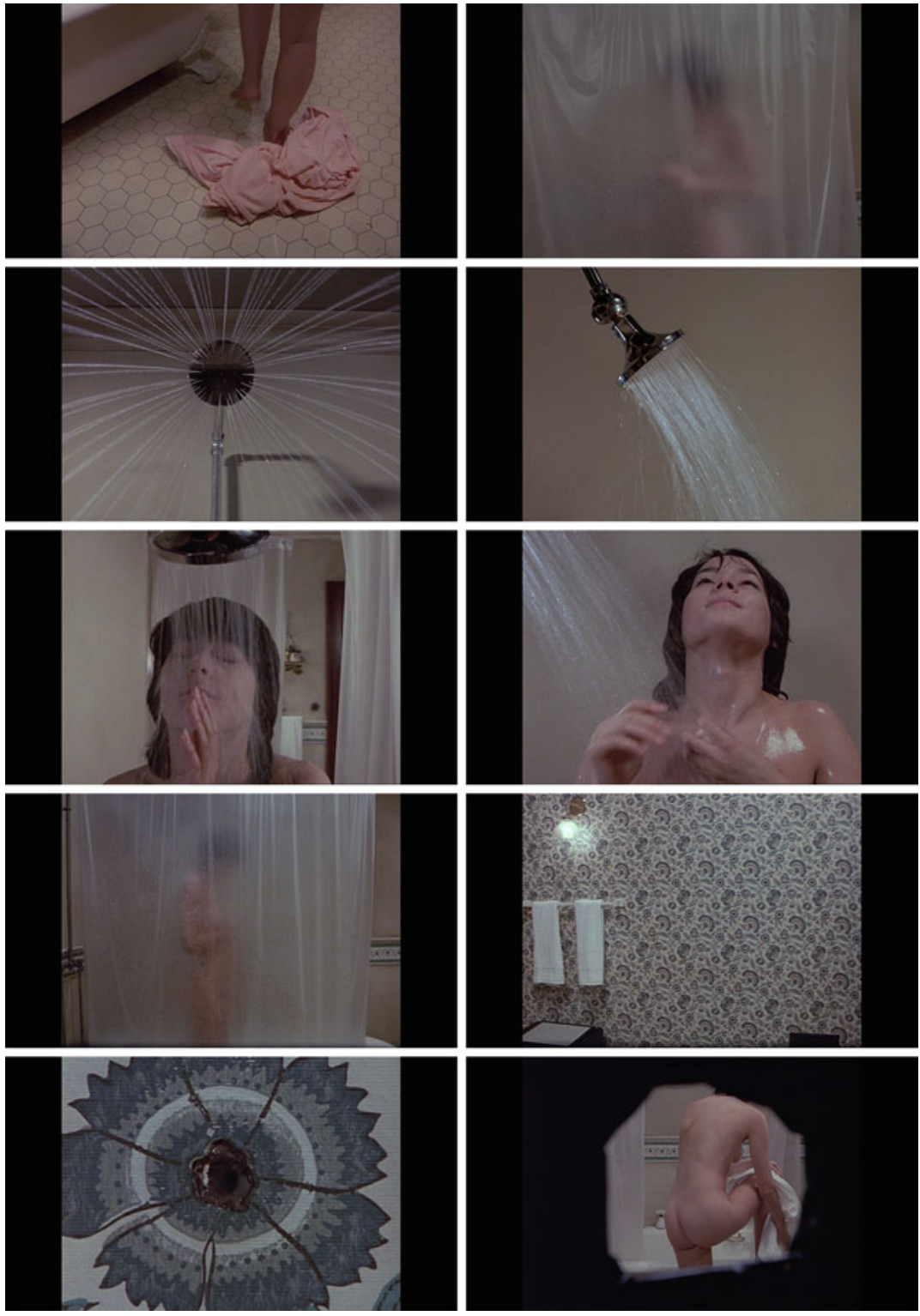

Abb.4.10a-j Psycho II, „Die Variation der Duschszene“, USA 1983 
hinter dem sie wie einst Marion schemenhaft zu erkennen bleibt. In der Dusche folgen seitliche und frontale Einstellungen des Duschkopfs sowie ihres Gesichts. Während Marion in Psycho nicht nur von vorn und von beiden Seiten gezeigt wird, sondern sich in einer langsamen Kamerabewegung auch die kommende Gefahr ankündigt, wechselt die Kamera in Psycho II wieder relativ schnell zurück auf den äußeren Blick auf den Duschvorhang, um ,neue 'Einstellungen folgen zu lassen (Abb. 4.10g-4.10j): Von der Dusche löst sich der Blick und dreht sich um 180 Grad entlang der eigenen Achse zur gegenüberliegenden Wand, um sich ähnlich der doppelten Annäherung von Gestalt und Kamera in Psycho langsam auf das Loch im floralen Tapetenmuster anzunähern. In diesem taucht überraschend ein anderes Auge auf. Die Kamera alterniert zwischen den gegenläufigen Blicken, bis Mary, Verdacht schöpfend, in Richtung des Gucklochs schaut. Nicht nur Mary vermutet, heimlich von Norman beobachtet zu werden; das Einstellungsarrangement legt auch kurzzeitig für die Zuschauenden nah, dass der voyeuristische Blick Norman gehört. Diese ,neue' Duschszene verkehrt Psycho auf mehreren Ebenen.

Innerhalb der intim-erotischen Rahmung, die Psycho II deutlich an gegenwärtige Darstellungskonventionen von Nacktheit anpasst, betritt Marion in Psycho noch einen geschützten Raum, in dem das brutale Ende noch nicht abzusehen ist und das gleichförmige Rauschen des Wassers eine sinnlich-berauschende Intensität erlaubt. Die Dusche markiert innerhalb des generischen Settings eines Slasherfilms inzwischen einen gefährlichen Ort des Alltäglichen, mit dem nicht nur eine antizipierte Angstlust einhergeht. In Psycho II unterläuft das (erinnerte) Wissen um genau diese trügerische Ruhe und ihr brutales Ende auch die Wahrnehmung der Szene. Die Autorität von Psycho's Duschszene determiniert Marys Duschszene von ihrem potentiellen Ende her. Während sich die ersten Einstellungen in der Dusche deutlich eng an Psycho orientieren und trotz der räumlichen Variation (Haus statt Motel) eine Parallelität aufrufen, deutet sich mit dem Wechsel zurück auf die Außenperspektive eine strukturelle Abweichung an. Doch in der langsamen Annäherung an das Guckloch und dem plötzlich auftauchenden Auge hält diese Abweichung die gleiche semantische Bedrohung bei, die über die doppelte Annäherung und theatrale Öffnung des Duschvorhangs in Psycho bewirkt wird. Auch in Psycho II wird kein furchterregendes Gesicht geboten, sondern nur eine , anonyme Horrorvision', deren Blick sich genauso an die Zuschauenden richtet wie die Attacke in Psycho. Während im Vergleich zu Psycho die Duschszene und der voyeuristische Blick verkehrt erscheinen, denn in Psycho geht Normans Blick der Duschszene voraus, wird dieser spezifische Zusammenhang in Psycho II widerlegt. Nicht nur hält sich Norman im Erdgeschoss auf; 
sein voyeuristischer Blick aus Psycho ist auch nicht Teil des unfinished business der Duschszene. Vielmehr wird die bleibende Gefahr über das generische Wissen ergänzt, dass das heimliche Beobachten nackter Körper potentielle Morde ankündigt und sexuell vorkonnotiert. Mary wird zwar in der Dusche nicht erstochen, ihr Tod scheint aber angesichts der bleibenden Gefahr ebenso nur aufgeschoben wie die genrespezifische ,Entladung' der aufgebauten Anspannung. In der Antizipation kommender Morde erhält die Anspannung bereits in der darauffolgenden Szene im zwielichtigen Motelmanager Toomey ihr erstes Opfer.

Die Blickanordnung und die Gefahr des anonymen Auges wird in Psycho II ein weiteres Mal ausgespielt, wenn Mary schließlich das Guckloch im Badezimmer entdeckt und selbst hindurch in das ehemalige Zimmer von Normans Mutter schaut (Abb. 4.11a-4.11c). Als sie das Versteck des Gucklochs auf der anderen Wandseite hinter einem Vogelbild entdeckt und von da selbst in das Badezimmer blickt, taucht wieder das ominöse Auge auf (Abb. 4.11d). ${ }^{29}$ Erneut zeigt sich die anonyme Horrorvision, deren mörderische Semantik und sexuelle Konnotation sich gegen die von Mary eingenommene voyeuristische Position wenden und ein Schockmoment produzieren, den die vorherige Duschszene noch nicht besaß. Wem das Auge gehört, bleibt bis zum Ende unklar. Während in Psycho die Blicke und Morde auf Normans psychotische Persönlichkeit zurück geführt werden, die über die destruktiv-monströse Semantik einer dominanten Mütterlichkeit gerahmt werden, übersetzt Psycho II dieses ödipale Konstrukt von „Mother“ in eine wahre monströse Mutterfigur, die sich als Ms. Spool aus dem Diner herausstellt. Während Psycho II als Nachfolgeproduktion transformativ auf Psycho zurückwirkt, führt die Duschszene aus Psycho zu einer genrespezifischen Lektüre der ,neuen' Duschszene, die von ihrem früheren Ende her unterlaufen wird und Erwartungen an ein Schreckensmoment weckt. Auch wenn Mary nicht zum neuen Dusch-Opfer wird, bleibt trotz der strukturellen Abweichungen eine bedrohliche Semantik bestehen, die allerdings in der Dusche keine Mordszene mehr generiert. Vielmehr wird sie genutzt, um über heimliche, erotische Blicke eine mörderische Instanz einzuführen und kommende Morde vorauszudeuten.

\footnotetext{
${ }^{29}$ Während das Guckloch in Psycho hinter einem Gemälde der Susanna im Bad versteckt ist und darin eine sexuell konnotierte Motivik festschreibt, ist diese inzwischen zur Genrekonvention geworden. Daher referiert Psycho II auch mit dem Vogelgemälde direkt auf die psychosexuelle Ausrichtung, die Psycho im Genre bewirkt hat.
} 

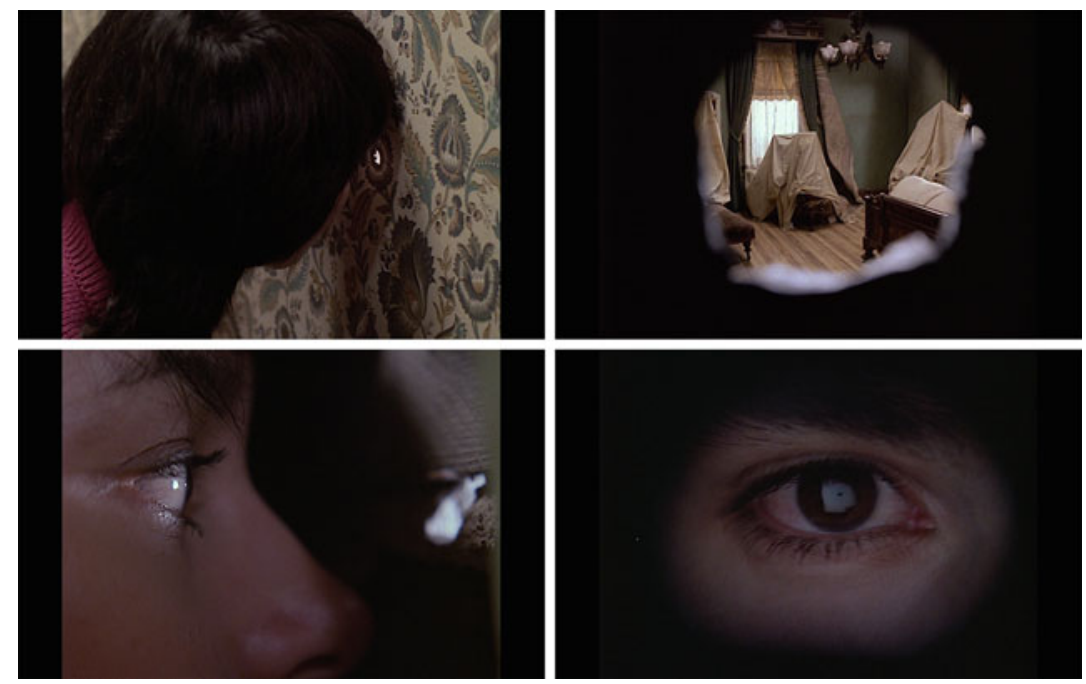

Abb.4.11a-d Psycho II, „Die Entdeckung des ,neuen“ Gucklochs“, USA 1983

In der strukturellen Veränderung nimmt Psycho II eine Distanzierung zu der Vorgängerproduktion vor und hebt die Wirkmechanik der Duschszene hervor. Diese erste strukturelle Distanzierung wird in der nachfolgenden Fortsetzung von Psycho III weiter ausgebaut, indem die Duschszene aus Psycho erneut aufgerufen, aber diesmal semantisch verkehrt wird.

\subsubsection{Das unüberwindbare Erinnerungsmoment in Psycho III}

Während Psycho II die Duschszene prominent an den Anfang stellt, fügt Psycho III die Duschszene als Flashback erst im Verlauf der Handlung ein. Während des Gesprächs mit der Reporterin Tracy Venable, die zu Normans Rehabilitation und dem Mord an Marion Crane recherchiert, beobachtet Norman, wie eine junge Frau (Maureen) einem Lastwagen entsteigt und das Diner betritt. Ihr Anblick löst in Norman Erinnerungen an den Duschmord aus. Auf die Fragen der Reporterin entgegnet Norman, dass ihn die Vergangenheit nicht loslässt: ,The past is never really past. It stays with me all the time. And no matter how hard I try; I can't really escape. It's always there, throbbing inside you, coloring your perceptions of the world, and sometimes controlling them." Zusammen mit dem 
Flashback des Muttermords aus Psycho II nutzt Psycho III hier typische Fortsetzungsstrategien, die Normans weitere Entwicklung in einen generisch-seriellen Sinnzusammenhang mit den vorherigen Produktionen stellen.

This kind of flashback is a typical strategy for sequels to refresh the viewers' memory. By evoking its two predecessors in this manner, the sequel visually reaffirms that all three movies function as installments of an ongoing narrative, and this retrospective serialization ultimately affects the ways in which audiences make sense of Norman Bates' actions in the earlier films. (Loock 82)

Bereits hier stellt Psycho III zwischen den ,befragten' Geschichten (Marion in Psycho und Lila in Psycho II) und der , neuen' Geschichte (Maureen in Psycho III) eine erste Verbindung her, die durch Normans ,the past is never really past“ selbstreflexiv markiert ist. Norman kann sich von den Fesseln seiner Vergangenheit nicht befreien und bleibt in Wiederholungsschleifen gefangen. So löst der Anblick der jungen Frau und der Initialien M.C. auf ihrem Koffer einen Flashback aus, der Norman Ausschnitte der Duschszene - ihren Schrei, die schwarze mordende Gestalt und die brutale Tötung (Abb. 4.12a-4.12d) - sehen lässt. Nur für einen kurzen Moment kehrt der Film in die Gegenwart des Diners zuvor, bevor weitere Bilder der Duschszene folgen, die zeigen wie Marion langsam an der Duschwand hinuntergleitet (Abb. 4.12e) und tot über den Badewannenrand fällt (Abb. 4.12f). Dabei löst sich Marions toter Blick (Abb. 4.12g) in eine farbige Einstellung auf, die unheilvoll den toten Blick von Maureen trägt (Abb. 4.12h). Von der auditiven Ebene und ihren messerscharfen ,Violinenschreien' sowie ihrem quälenden Horror befreit, schieben sich die Flashbacks wie visuelle ,Störungen“ ein. Während über den ersten Bildern noch die Fragen der Reporterin zu hören sind, erklingt über die zweite Sequenz nur noch das kratzende Geräusch einer stockenden Schallplatte, die immer wieder dieselben Töne anspielt.

Erneut gehen die Filme, wie bereits am Anfang von Psycho II, in einer gemeinsamen Aufnahme nahtlos ineinander über. Diese gemeinsame Aufnahme imitiert den toten Blick aus Psycho und nutz das Schwarzweiß, um die neue Aufnahme zu kaschieren. Erst der Farbwechsel löst die Einstellung als ,neu' und imaginativ auf. Ein weiteres Mal wird die Duschszene verändert und in ihrer szenischen Länge auf zwei Tötungsmomente - die Attacke und Marions Tod - reduziert. Während die Veränderungen in Psycho II die Duschszene zu einer (historisch geprägten) markanten Genresignifikanz verdichten, wird sie durch die fehlende Tonspur in ihrer raumgreifenden Wirkung beschnitten und als unüberwindbares Erinnerungsmoment, das Norman nicht los wird, eingeschoben (,It stays with me“). Auch in Psycho III markieren die Flashbacks Psycho und die Duschszene 

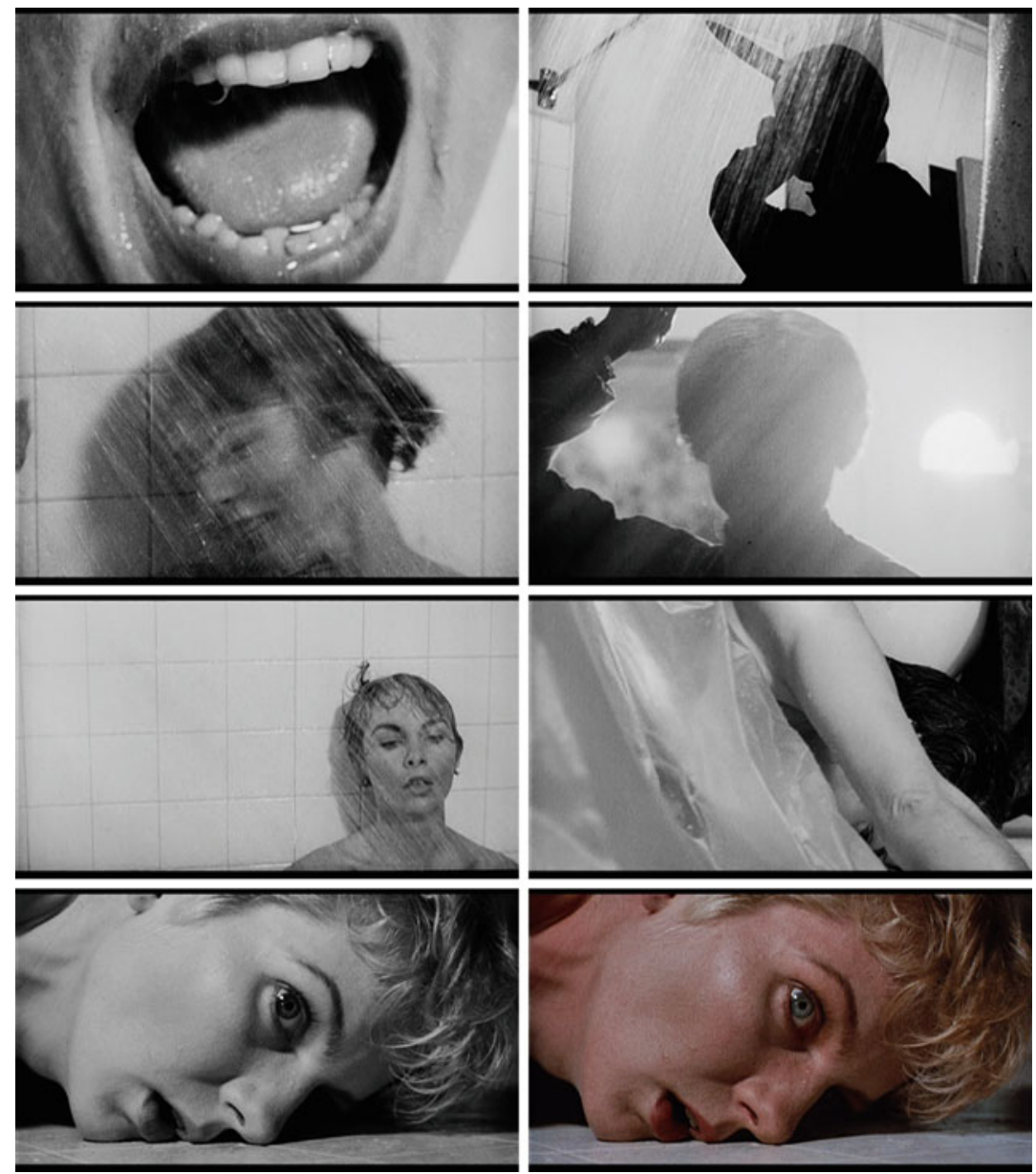

Abb. 4.12a-h Psycho III, „Die Duschszene als Flashback“, USA 1986

als vorausgehendes Bezugsobjekt, das seine erinnernde Semantik nachträglich über die Gestaltung als Flashback und die Vergegenwärtigung in Bezug auf Norman und Maureen erhält. Damit verschiebt Psycho III die generische Signifikanz, die die Duschszene auch in ihrer bereits historischen Prägung in Psycho II noch einnimmt, ganz in eine Erinnerungsignifikanz. 
In Normans Blick und der, gemeinsamen' Aufnahme bildet die Duschszene weiterhin ein unfinished business, das nicht nur Psycho nachträglich verändert, sondern auch die Fortsetzungsgeschichte von Psycho III determiniert und in Maureens (von Norman imaginiertem) toten Blick bereits ihren Tod ankündigt. Hierbei unterläuft die Duschszene nicht nur den weiteren Verlauf der Handlung, sondern zieht auch eine Lektüre von Maureen als Marions Doppelgängerin (M.C.) ein, die der bisherigen Erzählung nachträglich eine spezifische Ähnlichkeit zu Psycho zuschreibt: Auch Maureen bricht aus ihrem bisherigen (Kloster-) Leben aus und landet über Umwege im Bates Motel. Die Wege von Marion und Maureen sind in Psycho und Psycho III auf unterschiedliche Weise von ,fatalen' Ereignissen, Zweifeln und Verunsicherungen gepflastert. Während Marions Flucht zwar kriminell konnotiert ist (der Diebstahl von 40.000 Dollar), aber vor allem durch die Hoffnung auf eine, anständige' Zukunft erfolgt, flieht Maureen nach einem tragischen Unfall aus dem Kloster und vor ihren religiösen Zweifeln (,There is no God!“). Sie fliehen letztlich beide nur indirekt vor ihren vergangenen (kriminellen und tragischen) Taten, sondern hoffen vielmehr, woanders eine bessere Zukunft zu finden. Auf ihren Wegen begleitet die Figuren eine emotionale Verunsicherung und Desorientierung, die in den verregneten Windschutzscheiben und unklaren Blickverhältnissen ähnliche audiovisuelle Wahrnehmungsstrukturen erzeugen. Die Lichter entgegenkommender Autos und der Regen auf der Windschutzscheibe blenden auch Maureens Sicht; ihre inneren Unsicherheiten und Ängsten teilen die Zuschauenden wie einst mit Marion. Denn die regnerische Nacht kennzeichnet eine für Maureen unbekannte und düstere Welt außerhalb der Klostermauern. Während sich Marion von dem Polizisten verfolgt fühlt, stellt sich Maureens Mitfahrgelegenheit als eine gefährliche, zwielichtige Gestalt heraus, die sie mitten in der Nacht am Highway aussetzt. Letztlich finden beide im Bates Motel nach ihren individuellen Odysseen nur einen scheinbaren ,sicheren Hafen", denn Maureen ereilt ebenso das gleiche Schicksal - zwar nicht in der Dusche, aber im Haus wird sie ein Opfer von „Mother“. Im Motiv der Doppelgängerin erscheint zwar Psycho III als Wiederholung von Psycho, diese läuft aber in ihrer eigenen erzählerischen Singularität ab, wie sie Deleuze beschreibt. ${ }^{30}$ Dabei konkretisiert sich die grundsätzlich im Sequel angelegte Wiederholungsstruktur erst durch den Flashback der Duschszene zu einem zentralen Lektüremodus von Psycho III. Die erzählerische Engziehung offenbart wesentliche Differenzmomente, die sich erneut in einer Variation der Duschszene ausdrücken.

Mit Maureens Ankunft am Bates Motel und dem Bezug des Zimmers 1 wird eine weitere Duschszene auf narrativer Ebene aufgerufen, aber statt einer

${ }^{30}$ Zur Wiederholung bei Deleuze siehe auch Abschn. 2.2.4. 
strukturellen Abweichung wie in Psycho II verkehrt Psycho III die Duschszene semantisch in eine religiös konnotierte Errettungsvision. Norman betritt als „Mother“ mit der Intention, Maureen in der Dusche zu töten, das Motelzimmer. Darauf deuten nicht nur Details und Silhouette, sondern der Duschszene geht der voyeuristische Blick aus dem Hinterzimmer voraus, über den Norman einst Marion heimlich beim Auskleiden beobachtete und der auch den folgenden Mord(-versuch) sexuell konnotiert (Abb. 4.13a). ${ }^{31}$ Die in der Blickachse aufblitzende Messerklinge macht diese inzwischen paradigmatische Konnotation symbolisch manifest (Abb. 4.13b), sodass die folgende Duschszene nicht nur durch Psycho vorgeprägt ist, sondern auch der zur Genrekonvention gewordenen Verbindung von Sexualität und Gewalt folgt. Doch als sich der Duschvorhang öffnet und die dunkle Silhouette in bekannter Pose das Messer über Maureen erhebt (Abb. 4.13c), ergibt sich ein abweichendes Bild: Statt eines verängstigten und schreienden Opfers liegt Maureen mit aufgeschnittenen Pulsadern im blutgetränkten Wasser; im Nahtod erscheint ihr statt einer Horrorvision die Jungfrau Maria (Abb. 4.13d).
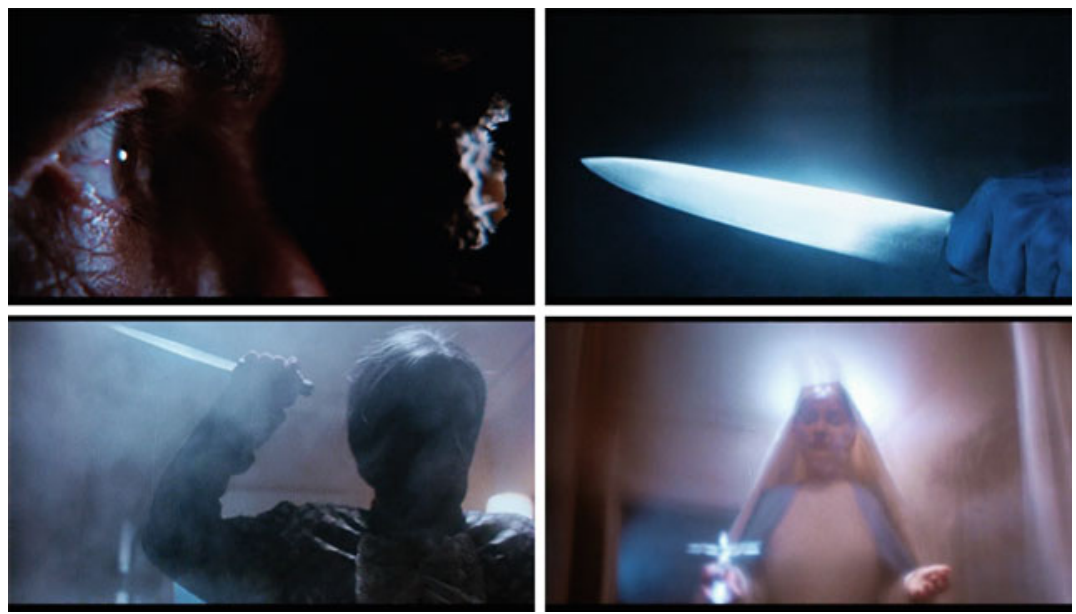

Abb. 4.13a-d Psycho III, „Die religiöse Umdeutung der Duschszene“, USA 1986

${ }^{31}$ Auch in Psycho III ist der Blick erotisch gerahmt und den Darstellungskonventionen von Nacktheit angepasst. Dabei wechselt der Blick wie in Psycho II kurzzeitig in das Motelzimmer, um eine gegenläufige Blickrichtung auf Normans Guckloch einzunehmen. 
Die religiöse Symbolik verkehrt die Alptraum-Metaphorik und zerstörerische Wirkung der Duschszene in eine ,Errettungsvision“, die erneut den erwarteten brutalen Mord in der Dusche verweigert. Maureens Tod ist ebenfalls nur aufgeschoben; auch sie kann dem Schicksal, dem die weiblichen Figuren in den Psycho-Sequels unterliegen, nicht entkommen und stürzt wie bereits der Psychiater in Psycho II (Abb. 4.14a) und der Privatdetektiv Arbogast in Psycho tödlich die Treppe hinunter (Abb. 4.14b). ${ }^{32}$ Die semantische Umdeutung der Duschszene folgt dem grundsätzlichen Differenzierungsbestreben der Sequels, sowohl in eine generisch-serielle Erzähleinheit mit Psycho aufzugehen, als auch sich zugleich von dem genreikonischen Status zu distanzieren. In Psycho III wird dies nicht nur über eine semantische Variation der Duschszene erzeugt, sondern auch über die Reduktion der Duschszene auf eine erinnernde Signifikanz, die als Flashback zudem dem Muttermord in Psycho II nachgeordnet wird.
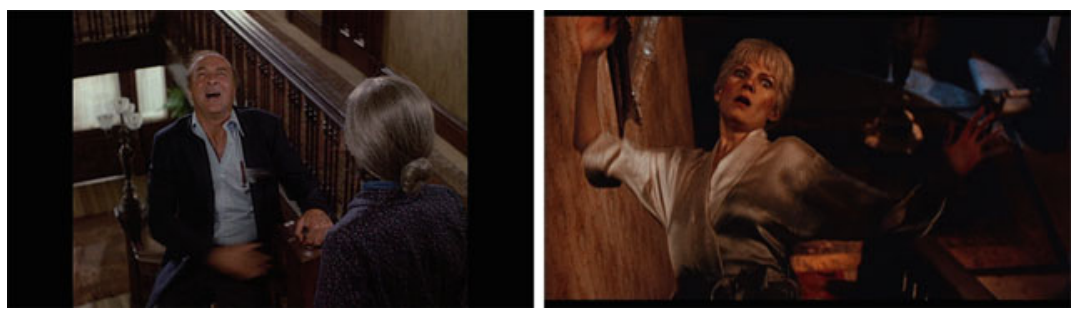

Abb. 4.14a und b Psycho II und Psycho III, ,Die Treppenmorde“, USA 1983 und 1986

In dem Umgang mit der Duschszene zeigt sich, wie sich die Sequels Psycho II und III wechselseitig in Bezug zu Psycho setzen und mit der Duschszene ein semantisch-medial präformiertes ,Genrezeugnis' aufgreifen, das sie als ,originären" Duschmord und unumgängliche Erinnerung nachträglich konstituieren und in Einstellungen imitieren (Haus und toter Blick). Zugleich wirken die Fortsetzungen darin transformativ auf Psycho zurück, indem sie die Erzählung nicht nur öffnen und fortführen, sondern auch verändern. Die Duschszene aus Psycho gerinnt innerhalb der Anordnungen zu einer selbstreflexiven Verbindungsstelle, die die Variationen einerseits als Schreckensmoment (Psycho II) und anderseits als Vorausdeutung (Psycho III) unterläuft. Doch die ,eigenen“ Versionen dekonstruieren gerade durch ihre strukturellen und semantischen Abweichungen die aufgerufenen Erwartungen und überführen die Duschszene in differente

${ }^{32}$ Sie stirbt dabei tragisch-ironisch durch Amors Pfeil, auf dem sie durch den Sturz landet. 
Versionen, die statt weiterer Dusch-Opfer neue (mörderische) Blickinstanzen (Mutterfigur in Psycho II) und neue Visionen (Errettung in Psycho III) hervorbringen. Dem ,primären“ Bezugsobjekt und seiner , historischen' Duschszene werden ,eigene" Kreationen gegenübergestellt, die in ihrer Differenzierung eine Loslösung von Psycho und einen Übergang in generisch-serielle Bezugsverhältnisse bewirken, in denen der Schrecken nicht mehr über die Duschszene, sondern über neue genrespezifische Momente eingezogen wird. $\mathrm{Zu}$ der so generierten , historischen' Signifikanz der Duschszene werden die neuen Morde der Sequels in ihren genrespezifischen Weiterentwicklungen und Darstellungskonventionen in Bezug gesetzt.

\subsection{Messer, Blut und Körper: Die neuen Tötungsszenen der Sequels}

Indem sich die wechselseitigen Relektüren der Sequels insbesondere an der Duschszene zuspitzen, diese als erinnerungswürdig herauslösen und als brutalen Duschmord wie unüberwindbares Erinnerungsmoment genreikonisch zu einer ,historischen' Genresignatur aufbauen, geraten dagegen die anderen Tötungsszenen aus Psycho, wie die Treppen- oder Kellerszene, in den Hintergrund. Zwar beziehen sich die Tötungsszenen in den Sequels auf den Treppenmord von Arbogast, indem sie den markanten Top Shot in der Wiederauferstehungsszene am Ende von Psycho II einsetzen oder die Fallperspektive bei den Tötungen des Psychiaters in Psycho II und von Maureen in Psycho III wiederkehrt. Ebenso findet sich die finale Attacke auf Lila ironisch gewendet in zwei Kellerszenen von Psycho II wieder, wenn Lila von ,Mother" ermordet wird und Mary als „Mother" verkleidet (Abb. 4.15a) und in der Pose aus Psycho von der Polizei erschossen wird (Abb. 4.15b). Aber die Sequels schreiben ihnen im Gegensatz zu Psycho's Duschszene keine prominente historische Genresignifikanz zu. Dem , einzigartigen“ Mord in der Dusche stehen in den Sequels zahlreiche neue Morde gegenüber, die sich aber nicht nur auf Psycho's Treppen- und Kellerszenen beziehen lassen. Für die auffällige Häufung an Tötungsszenen, vier bis sechs pro Sequel, muss ein neuer genrespezifischer Erwartungs- und Wertmaßstab herangezogen werden: Für das Publikum der Sequels, insbesondere im Kontext des Slasherfilms, zählt unter anderem der body count an getöteten Figuren, an dem , gemessen ' wird, ob ein Horrorfilm , abliefert'. Neben diesem pragmatischen Standard ist auch die Gewaltästhetik der neuen Tötungsszenen nicht mehr über die suggestive Montageästhetik von Psycho zu erklären, weil der Horror der Duschszene ,less in the actual images than in their summary implication“ auszumachen 
ist (Clover, „Men, Women“ 41). Die technische Weiterentwicklung von Special Effects hat im Horrorgenre als ,repositories of such effect“ (ebd. 41) seit Psycho eine neue Explizitheit hervorgebracht, die der Slasherfilm in der Verstümmelung und Zerstückelung von Körpern perfektioniert hat. Parallel zu der technischen Effektentwicklung sieht Clover auch ein gesteigertes Faszinosum für das damit einhergehende Öffnen von Körpern.

Beyond that, the slasher evinces a fascination with flesh or meat itself as that which is hidden from view. When the hitchhiker in Texas Chain Saw I slits open his hand for the thrill, the onlookers recoil in horror - all but Franklin, who seems fascinated by the realization that all that lies between the visible, knowable outside of the body and its secret insides is one thin membrane, protected only by a collective taboo against its violation. It is no surprise that the rise of the slasher film is concomitant with the development of special effects that let us see with our own eyes the ,opened' body. (Ebd. 32)
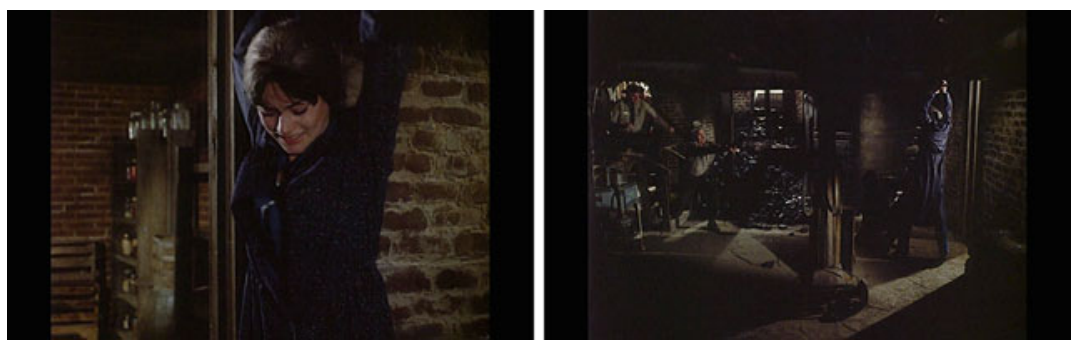

Abb. 4.15a und b Psycho II, „Mary als Mother verkleidet“, USA 1983

Das Öffnen der Körper verlangt im Genre eine spezifische Waffenart, die Clover als ,pretechnical“ (ebd. 31) bezeichnet: Nahkampfwaffen wie Messer, Hammer, Axt, Eispickel oder Kettensägen erlauben gegenüber Schusswaffen, Pfeil und Bogen oder Katapulten eine schnelle und leichte Handhabung, aber insbesondere führen sie in einer ,primitive, animalistic embrace“ (ebd. 32) näher an die Opfer heran. Diese körperliche Komponente der Waffen ist generisch nicht zu unterschätzen. In der Hand der Angreifenden erweitern sie den Körper um einen 
symbolischen Phallus, den sich Normans weiblich konnotierte Identität als „Mother" erst aneignet, ehe sie ihre Opfer terrorisiert. ${ }^{33}$ Den meisten Tötungsszenen geht daher der Griff zum Messer voraus, die das Töten ankündigt. ${ }^{34}$ Bevor jedoch die Körper malträtiert werden (können), wird das Messer in seiner phallischmörderischen Symbolik nicht nur blitzend in die Höhe gehalten, sondern auch von entsetzten Blicken und schreienden Gesichtern in seiner Symbolik ,anerkennend wahrgenommen (Abb. 4.16a-4.16d). ${ }^{35}$ Das Messer strukturiert auf audiovisueller Ebene die Affektdramaturgien der Tötungsszenen: Es kündigt die Tötung an und erzeugt über die auslösenden Schreie der Opfer (und des Publikums) einen affektiven Spannungshöhepunkt, bevor es in die Körper eindringt und tiefe Einstiche und Schnitte in den Bäuchen, Brüsten und Gesichtern hinterlässt.
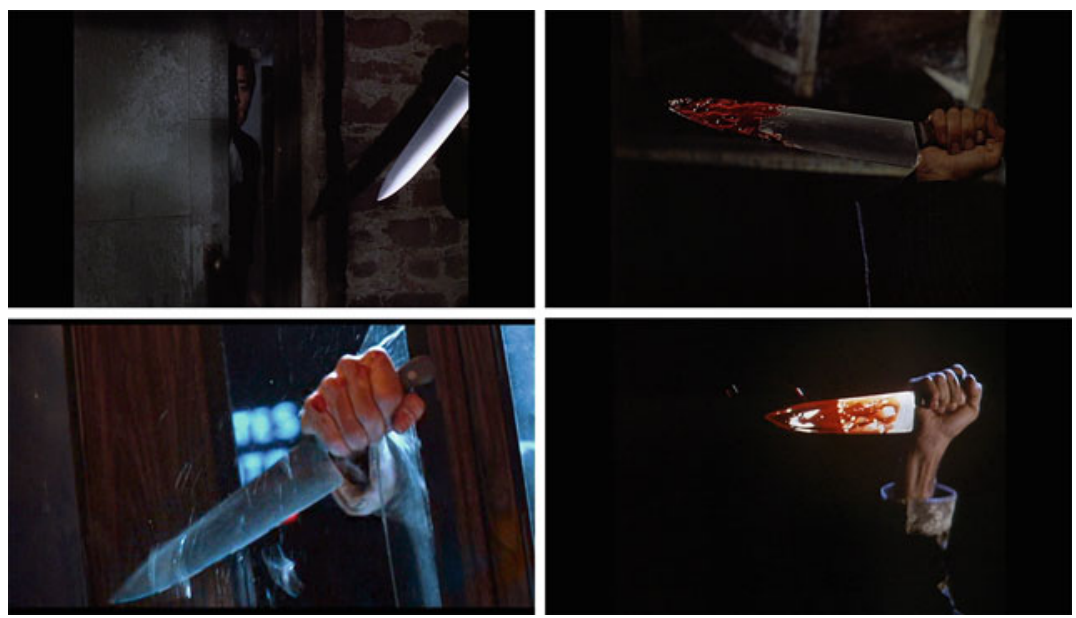

Abb. 4.16a-d Psycho II-IV, „Das Messer“, USA 1983, 1986 und 1990

\footnotetext{
${ }^{33}$ Insbesondere für Clovers Konzept des Final Girl und ihre Transformation vom Opfer zur zurückschlagenden Heldin ist diese phallische Komponente bedeutsam (,Men, Women“ 35-41).

${ }^{34}$ Das Messer gilt durchaus als generischer Regelfall, auch wenn die Sequels die Tatwaffen variieren und ebenso Strangulationen (Psycho IV), Vergiftungen (Psycho III und Psycho IV) und Affekthandlungen (Psycho II und Psycho III) nutzen.

${ }^{35}$ Zur phallischen Symbolik siehe Williams, „Film Bodies“.
} 
Für den Slasherfilm gerinnt das Messer in seiner phallischen Symbolik und zerstörerisch-exzessiven Körperästhetik zu einer übergreifenden Genre-Chiffre. Entgegen der suggestiven Montage und Schwarzweiß-Ästhetik von Psycho inszenieren die Sequels über nackte, schreiende, durchstochene und blutende Körper eine ekstatische Spektakularität, wie sie Williams in ihrem Konzept der body genres beschreibt („Film Bodies“). Diese arbeitet am Körper der Zuschauenden und affiziert die (Körper-)Wahrnehmung der Zuschauenden. Gerade weil in den Sequels Gewalt nicht mehr nur suggeriert, sondern explizit gemacht wird, schwindet die ästhetische Distanz zur Darstellung und die Zuschauenden erleben eine sadistisch-masochistische Angstlust. Dies äußerst sich für Clover in einer ebenso exzessiven Erlebnisform: „More particularly: spectators tend to be silent during the stalking scenes (although they sometimes call out warnings to the stalked person), scream out at the first slash, and make loud noises of revulsion at the sight of the bloody stump.“ (Clover, „Men, Women“ 41) Die genrespezifischen Affektdramaturgien sind dabei vor allem entlang der weiblichen Opfer und ihrer Körper angelegt, auch wenn es im Slasherfilm wie in den Sequels weibliche und männliche Opfer gibt und beide eine gemeinsame ,Sündhaftigkeit' kennzeichnet: „Kiling those who seek or engage in unauthorized sex amounts to a generic imperative of the slasher film. It is an imperative that crosses gender lines, affecting males as well as females." (Ebd. 34) Als sich das junge Pärchen in Psycho II heimlich in den Keller schleicht, wird es von „Mother“ präkoital überrascht. Während das Mädchen noch fliehen kann, wird der Junge niedergestochen. Während seine Tötung neben einiger Einstiche vornehmlich über suggestive Bilder (Messer, zersplitternde Konserven und Hand) wesentlich ,geschlechtsneutraler ${ }^{\text {" }}$ verläuft, sterben die weiblichen Opfer in Psycho III einen postkoitalen und blutigen Tod (Abb. 4.17a und 4.17b). Auch wenn die Motelmanager in Psycho II und Psycho III eine zwielichtige ,Sündhaftigkeit' teilen, die sie letztlich ihren Tod kostet, differenzieren die Darstellungen deutlich zwischen den Geschlechtern. Die weiblichen Opfer erleiden nicht nur einen detail- wie variantenreicheren Tod; an ihren Körpern werden die Gewaltfantasien in ihrer sexuellen Konnotation wesentlich expliziter ausgelebt. Den Tötungsszenen geht, wie bereits bei Marion in Psycho, einerseits eine sexualisierte Ausstellung ihrer Körper über voyeuristische Blicke, heimliche Treffen oder Sexszenen voraus, anderseits begleiten ihre Todessschreie die ,Stabbing '-Sequenzen stets lautstark über Großaufnahmen ihrer aufgerissenen Münder. 

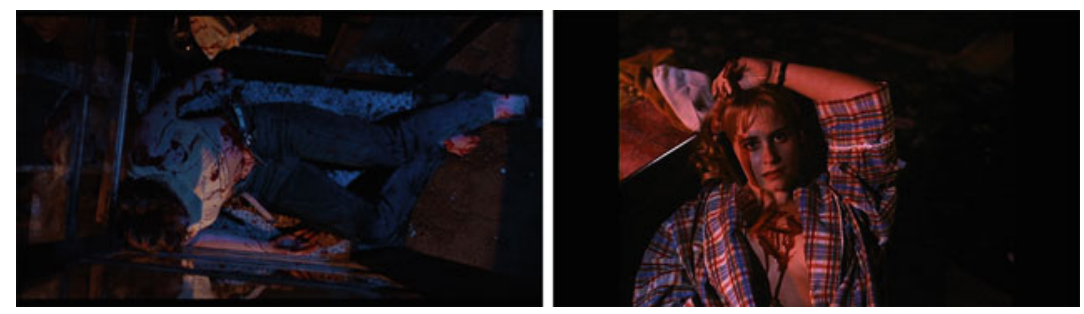

Abb.4.17a und b Psycho III und IV, „Geschlechterdifferenz“, USA 1986 und 1990

Ihre Sexualität erhält allerdings keine, verdiente“ Bestrafung, sondern birgt ein spezifisches Genrevergnügen, wie Williams unter anderem in ihrem Konzept der body genres betont (,Film Bodies“). Über nackte Körper und heimliche Blicke stellen auch die Sequels eine Sexualität auf sensationelle Weise zur Schau, die an kulturellen Tabuvorstellungen rührt und Sex verhängnisvoll mit expliziten Gewaltfantasien und sexuell uneindeutigen Konnotationen verknüpft, um ein transgressives Gewalt- und Geschlechtererleben zu generieren (Williams, „Discipline and fun“ 361 f.). Das heißt, dass Sexualität im Horrorgenre weniger als Kontrastfolie als vielmehr als gemeinsames Fantasieerleben angelegt ist. An den ,neuen“ Tötungsszenen wird besonders deutlich, wie in den Sequels die genreund genderspezifischen Darstellungskonventionen des Slasher- und Horrorfilms einfließen und nicht nur die neuen technischen Möglichkeiten der Special Effects genutzt werden, sondern auch die sexualisierten Gewaltfantasien vor allem an den weiblichen Körpern operieren. Im Vergleich zu Psycho bieten die Sequels in ihrer expliziten Ästhetik eine eindeutigere Horrorqualität an.

With this new explicitness also comes a new tone. If the horror of Psycho was taken seriously, the ,horror" of the slasher films is of rather more complicated sort. Audiences express uproarious disgust (,Gross!“) as often as they express fear, and it is clear that the makers of slasher films pursue the combination. (Clover, „Men, Women“ 41)

Zwar weisen die Fortsetzungsmorde im Vergleich $\mathrm{zu}$ dem ,historischen“ Duschmord eine explizitere Gewaltästhetik auf, aber die Szenen in den Sequels wie in Psycho operieren vor dem Hintergrund ihrer Zeit mit denen ihnen zur Verfügung stehenden genrespezifischen Affektdramaturgien und erzeugen ihre Schockwirkungen jeweils über einen körperbetonten Schrecken. Bereits in der Duschszene ist die ausgestellte Nacktheit von Marion sexuell konnotiert und mit 
suggerierten Gewaltdarstellungen verbunden, die die Sequels mit dem technischen Fortschritt der Special Effects in eine explizite Form übersetzen und in Farbe zeigen. Die ,neuen“ Tötungsszenen fügen sich damit in das übergreifende semantische Konzept des Slashergenres ein und liefern einen spezifischen Schrecken ihrer Zeit. Inwiefern diese genrespezifische Konventionalität der Sequels angesichts der wechselseitigen Relektüren zu Psycho genrehistorisch bedeutsam wird, wird abschließend diskutiert.

\subsection{Die generisch-seriellen Interdependenzen als generationenübergreifende Horrorversion}

In den Sequels entsteht ein wechselseitiges Verbindungsgeflecht, das Psycho nicht nur in eine Fortsetzungserzählung überführt, sondern die Geschichte im Kontext des Slasher- und Horrorgenres fortsetzt und die seriellen Variationen der Figuren, Orte und Tötungsszenen auf eine gemeinsame Genresemantik engführt. Während Psycho II das Motiv der destruktiv-monströsen Mutter in einer tatsächlich mordenden Mutterfigur konkretisiert, ist diese in Psycho III verantwortlich dafür, dass Normans mörderisches Alter Ego „Mother“ wiederaufersteht und sowohl in ihrer stimmlichen Dominanz als auch in ihrer mumifizierten Erscheinung zu einer wiederkehrenden Horrorattraktion wird. Die übergreifende ödipale Semantik wird zusätzlich in Psycho IV auf ein inzestuöses Verhältnis zur Mutter zurückgeführt. Im Rahmen dieses zugespitzten Erklärungsmusters bleibt Norman Bates in den Sequels trotz der sichtbaren Alterung von Anthony Perkins weiterhin das unschuldig-naive Opfer, das der Zwangsläufigkeit seines psychopathologischen Rückfalls und seiner Wiederholungstaten in den Sequels nicht entkommen kann. Dieser generisch-seriellen Unausweichlichkeit unterstehen auch Haus und Motel, die nicht nur (weiterhin) Raum und Zeit merkwürdig entrückt wirken, sondern inzwischen auch von der kulturellen Semantik der realweltlichen Orte selbst überholt werden. Dennoch werden Haus und Motel in den Sequels unentwegt als Schreckensorte reinszeniert. Während die Figuren und Orte damit von Psycho gelöst und in neue generisch-serielle Erzählzusammenhänge überführt werden, gestaltet sich der Umgang mit der Duschszene in den Sequels anders.

Über die Duschszene binden die ersten beiden Sequels Psycho als einen, originären' Horrorfilm ein, wobei sie seinen generischen Status erst nachträglich über signifikante Veränderungen (Kürzungen und Tonbeschneidungen) und markante Positionierungen als Auftakt und Flashback selbst herstellen. Die Duschszene wird damit auf ihre Genremarkierung als Horrormoment und Erinnerungsmoment komprimiert, sodass andere Markierungen wie Semantiken, die Psycho als 
einen dynamischen und variablen generischen Text kennzeichnen, ausgeblendet werden. Als Zitat ist die Duschszene jedoch semantisch-medial präformiert und von einer diskursiven Historizität durchzogen, die dem Schrecken eine historische Prägung verleiht und genrespezifisch nicht mehr erlebbar wird. Als ,historischer Duschmord bleibt sie jedoch weiterhin wirkungsvoll. Die bedrohliche Semantik und der szenische Verlauf unterlaufen die ,neuen' Duschszenen von ihrem potentiellen Ende her, wobei die Sequels in der Variation dieses Endes genau diese Semantik und Szenerie dekonstruieren und keine neuen Dusch-Opfer anbieten. Stattdessen führen sie eine neue mörderische Blickinstanz ein (Psycho II) und verkehren die Horrorvision in eine ,Errettungsvision' (Psycho III). Während die Variationen die Erwartung auflösen, dass sich in der Dusche erneut Tötungen ereignen, schreiben sie wiederum die Duschszene als eine ,historische" Genresignatur von Psycho fest, deren einziges Dusch-Opfer Marion bleibt. Die Sequels finden dennoch zahlreiche neue Opfer, die in eine abwechslungsreiche Serienmordreihe eingehen und entlang der Darstellungskonventionen des Slashergenres inszeniert werden. Als historische Genresignatur setzen die Sequels die Duschszene allerdings weniger an den Anfang dieser neuen Reihe brutaler Serienmorde, sondern bieten sie vielmehr dem eigenen Genrestatus als Slasherkorpus zum Vergleich an und demonstrieren demgegenüber ein ausgeprägtes Genrevergnügen, das die sexuell konnotierten Gewaltfantasien des Genres an nackten und malträtierten Körpern auslebt. Die Sequels ziehen hier einen Vergleich, der ihre eigene Gewaltästhetik verstärkt. Aus einer genrehistorischen Perspektive aber folgen die Sequels der gleichen Affektstrategie wie Psycho, die expliziteren Darstellungen rühren dagegen aus den technischen Weiterentwicklungen der Special Effects her.

Die Sequels greifen letztlich nicht nur vertraute Figuren, Orte und Szenen auf und führen sie als Slashervariationen fort, vielmehr wirken sie gerade durch die selbstreflexive Perspektivierung der Duschszenen an der eigenen Genrehistoriografie mit. Einerseits positionieren sie sich dadurch deutlich zu Psycho, indem sie die Produktion nicht nur nachträglich als vorgängig markieren, sondern darin semantisch verschieben und als Horrorikone festschreiben. Anderseits gestalten die Sequels daran anschließend ihre Fortführungen entlang von Slasherkonventionen und führen die Variationen auf eine gemeinsame Horrorsemantik eng, die durch den Vergleich zu Psycho umso deutlicher in ihrer genrespezifischen Weiterentwicklung sichtbar wird. Diese wechselseitigen Relationierungen kehren die Verhältnisse zwischen Primärem und Sekundärem um, denn es sind die Sequels, die Psycho im Horrorgenre derart signifikant verorten und zu ihrer eigenen Genrekonventionalität in Bezug setzen. In der iterativen Fortführung lösen die Sequels Norman, „Mother“ und die Orte von Psycho ab und entwickeln sie zu dynamischen Genremerkmalen, deren ästhetische Variationen sich innerhalb 
einer spezifischen Genresemantik bewegen. Im Rahmen dieser neuen generischseriellen Zusammenhänge werden sie als Genresignaturen auf die Ebene des Genrekonzepts verschoben, ohne dass sie ihren Bezug zu Psycho verlieren. Vielmehr funktionieren sie weiterhin auf dessen Ebene und kennzeichnen den generischen Text in seiner semantischen Vielfalt. Aber zugleich haben sie eine weitere semantische Funktion angenommen und markieren ebenso den seriellgenerischen Intertext der Sequels als ,exklusives' Slasherkorpus. Die Perspektive auf die Iterationen erlaubt es auch, in den Sequels die diskursive Beschaffenheit von Psycho einzubinden, ohne der Analyse zuwiderzulaufen, sondern stattdessen produktiv zu vertiefen. Denn erst die enge intertextuelle Analyse der Iterationsmuster und Genrekonventionen verdeutlicht, wie sich die wechselseitigen Relektüren auf Psycho's diskursiven Status und auf die generisch-serielle Diskursivierung der Sequels auswirken und eine Doppelperspektive auf die Ebene von Genreproduktion und Genregruppierung ermöglichen. Auf beiden Ebenen lassen sich die Iterationen als dynamische Genresignaturen in ihrer diskursiven wie historisch komplexen Verfasstheit erfassen.

Auf der Ebene des Genrekonzepts bilden die Sequels schließlich eine Genregruppierung, die nicht einfach eine neue Psycho-Geschichte liefert, sondern durch die generisch-seriellen Variationen und semantischen Engführungen eine spezifische Horrorvision kreiert. Diese hält nicht nur Psycho im kulturellen Gedächtnis lebendig, sondern ergänzt die Genrehistoriografie um eine eindeutige Version, die in ihrer forcierten Semantik und aktualisierten Ästhetik ein erneutes Genrevergnügen bereitet. Die einzelnen Produktionen stehen also nicht nur in einer intertextuellen sowie generisch-seriellen Perspektive zueinander, sondern prägen auch ein generationenübergreifendes Genregedächtnis, in dem Psycho und die Sequels nicht nur spezifische Versionen ihrer Zeit bilden, sondern auch den jeweiligen Generationen gegenseitig verfügbar gemacht werden. Im Umgang mit medialen Texten und kulturellem Wissen nehmen die Fortsetzungen eine wichtige vermittlerische Brückenposition ein, die historische Elemente ,bewahrt', Vergleiche herstellt und Weiterentwicklungen aufdeckt. Während die neue Slashergeneration für die historische Signifikanz von Psycho sensibilisiert wird, wird eine ältere Psycho-Generation auf die explizitere Horrorsignifikanz der Sequels aufmerksam gemacht. Auf dieser generationenübergreifenden Genreebene bildet die jeweilige Version den Ausgangspunkt, von dem die anderen Produktionen erst in Bezug gesetzt werden. Für die ,zweite" Generation der Sequels wird Psycho zu einem historischen Referenzpunkt sekundärer Natur, während für die 1950/60er Generation von Psycho die Sequels nachfolgende Versionen darstellen. Die wechselseitigen Relektüren zwischen den Sequels und Psycho hebeln die zeitliche Ordnung aus ,Früher' und ,Später' aus und ziehen die Versionen 
in einer gemeinsamen Lektüre zusammen, sodass sich Vergangenheit, Gegenwart und Zukunft übereinanderschichten. Im Angesicht dieser ,neuen' Anordnungsverhältnisse, die der zeitlichen Produktionschronologie und der normativen ,Original'-Konstruktion entgegenlaufen, bilden die Iterationen nicht nur zwischen Psycho und den Sequels, sondern auch zur Prequel-Serie Bates Motel, eine generationenübergreifende, eigene Genregeschichte aus, die sich stetig fortschreibt, überschreibt und Genre- wie Zeitebenen übereinanderschichtet. Inwiefern diese Genregeschichte in Bates Motel durch das zeitgenössische Prequel-Konzept eine gesteigerte Komplexität annimmt, wird im folgenden Kapitel dargelegt.

Open Access Dieses Kapitel wird unter der Creative Commons Namensnennung 4.0 International Lizenz (http://creativecommons.org/licenses/by/4.0/deed.de) veröffentlicht, welche die Nutzung, Vervielfältigung, Bearbeitung, Verbreitung und Wiedergabe in jeglichem Medium und Format erlaubt, sofern Sie den/die ursprünglichen Autor(en) und die Quelle ordnungsgemäß nennen, einen Link zur Creative Commons Lizenz beifügen und angeben, ob Änderungen vorgenommen wurden.

Die in diesem Kapitel enthaltenen Bilder und sonstiges Drittmaterial unterliegen ebenfalls der genannten Creative Commons Lizenz, sofern sich aus der Abbildungslegende nichts anderes ergibt. Sofern das betreffende Material nicht unter der genannten Creative Commons Lizenz steht und die betreffende Handlung nicht nach gesetzlichen Vorschriften erlaubt ist, ist für die oben aufgeführten Weiterverwendungen des Materials die Einwilligung des jeweiligen Rechteinhabers einzuholen.

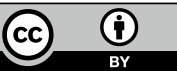

